\title{
Performance evaluation for inerter-based dynamic vibration absorbers
}

\author{
Yinlong $\mathrm{Hu}^{\mathrm{a}}$, Michael Z. Q. Chen ${ }^{\mathrm{b}, *}$ \\ ${ }^{a}$ School of Automation, Nanjing University of Science and Technology, Nanjing, China \\ ${ }^{b}$ Department of Mechanical Engineering, The University of Hong Kong, Hong Kong.
}

\begin{abstract}
This paper is concerned with the $H_{\infty}$ and $H_{2}$ optimization problem for inerter-based dynamic vibration absorbers (IDVAs). The proposed IDVAs are obtained by replacing the damper in the traditional dynamic vibration absorber (TDVA) with some inerter-based mechanical networks. It is demonstrated in this paper that adding one inerter alone to the TDVA provides no benefits for the $H_{\infty}$ performance and negligible improvement (less than $0.32 \%$ improvement over the TDVA when the mass ratio less than 1) for the $H_{2}$ performance. This implies the necessity of introducing another degree of freedom (element) together with inerter to the TDVA. Therefore, four different IDVAs are proposed by adding an inerter together with a spring to the TDVA, and significant improvement for both the $H_{\infty}$ and $H_{2}$ performances is obtained. Numerical simulations in dimensionless form show that more than $20 \%$ and $10 \%$ improvement can be obtained for the $H_{\infty}$ and $H_{2}$ performances, respectively. Besides, for the $H_{\infty}$ performance, the effective frequency band can be further widened by using inerter.
\end{abstract}

Keywords: Inerter, dynamic vibration absorber, $H_{\infty}$ optimization, $H_{2}$ optimization.

\section{Introduction}


solutions were analytically derived in [4] and it was also shown that the fixed-point method actually yielded an approximate but highly precise solution (with less than $0.5 \%$ deviation when the mass ratio less than 1). Another common performance measure of tuning DVA is the $H_{2}$ performance measure, which is desirable when the primary system subjected to random excitations. The objective of $H_{2}$ optimization is to optimize the total vibration energy of the system over all frequencies [5]. For the TDVA with undamped primary systems, the optimal tuning frequency and damping ratio were investigated in [5], and then the analytical solutions were derived in [6]. For damped primary systems, various design methods and tuning criteria have been proposed, such as those in $[7,9,8,10]$, and the applications of the TDVA in nonlinear and distributed primary systems have been investigated $[11,12,13]$. The active DVAs utilizing feedback control actions have also been proposed $[14,15,16]$.

Inerter is a two-terminal mechanical device proposed by Smith in 2002 [17], which has the property that the applied force at its two terminals is proportional to the relative acceleration between them and the constant of proportionality is called inertance with a unit of kilogram. Inerter has been successfully applied in Formula One racing car suspension systems [18], and now, applications of inerter in various mechanical systems, such as vehicle suspensions [19, 20, 21, 22] and vibration suppression systems [23, 24, 25, 26], have been investigated. Recently, the reduction of vibration systems' natural frequencies by using inerter has been theoretically demonstrated [27], and the interest in passive network synthesis has also been rekindled [28, 29, 30, 31, 32, 33, 34, 35, 38].

Vibration absorption is one of the potential applications of inerter [17]. In [17], the problem of designing inerter-based networks to absorb vibration at a specific frequency was studied. Thereafter, the suppression of vibration over a broadband frequency by using inerter has been proposed. In [23], an inerter-based configuration ( $C 4$ in this paper) was employed between adjacent storeys to suppress the vibration of a multi-storey building. In [24], optimal solutions for several inerter-based isolators (including all the configurations except $C 5$ in this paper) were algebraically derived based on a "uni-axial" vibration isolation system. In [25], a new configuration incorporating an inerter was proposed and applied to a mechanical cascaded (chain-like) systems. In [26], the dynamics of a tuned mass absorber with an additional viscous damper and an inerter attached to the pendulum was investigated.

In this paper, a novel structure for inerter-based DVAs (IDVAs) is proposed by replacing the damper in the TDVA with some inerter-based mechanical networks, and both the $H_{\infty}$ and $\mathrm{H}_{2}$ performances of the proposed IDVAs are investigated. It is demonstrated in this paper that adding an inerter alone to the TDVA, no matter it is in parallel connection or in series connection, provides no benefits for the $H_{\infty}$ performance and negligible benefits (less than $0.32 \%$ improvement over the TDVA when the mass ratio less than 1) for the $H_{2}$ performance. In contrast, by adding an inerter together with a spring to the TDVA (e.g. $C 3$, $C 4, C 5$, and $C 6$ in this paper), both $H_{\infty}$ and $H_{2}$ performances can be significantly improved. Over $20 \%$ improvement compared with the TDVA can be obtained for the $H_{\infty}$ performance, and the effective frequency band can also be further widened by using inerter. For the $\mathrm{H}_{2}$ performance, it is analytically demonstrated that the IDVAs proposed in this paper perform surely better than the TDVA and over $10 \%$ improvement is obtained in numerical simulation. Moreover, a minmax framework directly using the resonance frequencies is proposed for the $H_{\infty}$ optimization, and an algebraic method to analytically calculate the $H_{2}$ norm is employed for the $\mathrm{H}_{2}$ optimization. All these constitute the main contributions of this paper. 
The organization of this paper is as follows. In Section 2, the IDVAs in this paper are introduced and the dimensionless representations of displacement transfer functions are derived. In Section 3 and Section 4 , the $H_{\infty}$ and $H_{2}$ optimization problems are solved for the IDVAs and the comparison between the IDVAs and the TDVA is conducted. Conclusions are drawn in Section 5.

\section{Inerter-based dynamic vibration absorbers}

Fig. 1 shows the comparison between the IDVAs proposed in this paper and the TDVA, where the IDVA is obtained by replacing the damper in the TDVA with some inerter-based mechanical networks. The entire networks employed in this paper are shown in Fig. 2. The equations of motion for the whole system in the Laplace domain are

$$
\begin{aligned}
M s^{2} x & =F+F_{d}-K x \\
m s^{2} x_{a} & =-F_{d}, \\
F_{d} & =(k+s Y(s))\left(x_{a}-x\right),
\end{aligned}
$$

where $Y(s)$ is the admittance of the inerter-based passive mechanical networks and $F_{d}$ is the force of the DVA imposed on the primary mass $M$.

From (2) and (3), one obtains,

$$
F_{d}=-R(s) x
$$

where

$$
R(s)=\frac{(k+s Y(s)) m s^{2}}{k+m s^{2}+s Y(s)} .
$$

Then, one obtains the displacement transfer function as

$$
H(s)=\frac{x}{x_{s}}=\frac{1}{\frac{s^{2}}{\omega_{n}^{2}}+\frac{1}{K} R(s)+1},
$$

75 where $x_{s}=F / K$ and $\omega_{n}=\sqrt{\frac{K}{M}}$ are the static displacement and natural frequency of the primary system, respectively.

The admittance of each network in Fig. 2 is shown in Table 1 , where $Y_{i}(s), i=1, \ldots, 6$ corresponds to $C_{i}, i=1, \ldots, 6$ in Fig. 2, respectively. Substituting each $Y_{i}(s)$ into (4), one can obtain the detailed transfer function for each configuration. To obtain the dimensionless representation of each configuration, the following dimensionless parameters are defined as

$$
\left.\begin{array}{c}
\mu=\frac{m}{M}: \text { mass ratio } \\
\delta=\frac{b}{m}: \text { inertance-to-mass ratio } \\
\zeta=\frac{c}{2 \sqrt{m k}}: \text { damping ratio } \\
\eta=\frac{\omega_{b}}{\omega_{m}}: \text { corner frequency ratio } \\
\gamma=\frac{\omega_{m}}{\omega_{n}}: \text { natural frequency ratio } \\
\lambda=\frac{\omega}{\omega_{n}}: \text { forced frequency ratio }
\end{array}\right\}
$$




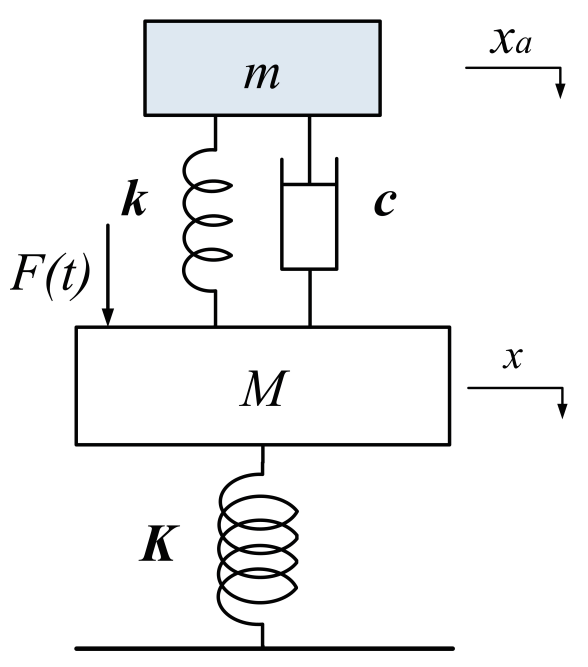

(a)

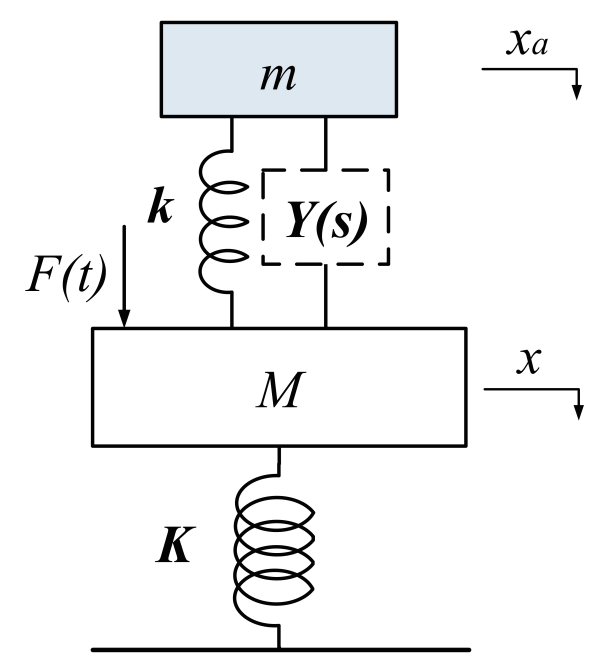

(b)

Figure 1: Dynamic vibration absorbers (DVA): (a) traditional dynamic vibration absorber (TDVA); (b) inerter-based dynamic vibration absorber (IDVA).

where

$$
\left.\begin{array}{c}
\omega_{m}=\sqrt{\frac{k}{m}}: \text { natural frequency of the DVA } \\
\omega_{b}=\sqrt{\frac{k_{1}}{b}}: \text { corner frequency of the DVA } \\
\omega_{n}=\sqrt{\frac{K}{M}}: \text { natural frequency of the primary system }
\end{array}\right\}
$$

Remark 1. In this paper, the force-current analogy between mechanical and electrical networks is employed, and admittance is defined to be the ratio of force to velocity, which agrees with the usual electrical terminology (see [17] for details). Such a definition is consistent with some books [36, p. 328], but not others which use the force-voltage analogy [37].

Remark 2. Since the natural frequencies would be perturbed by using inerter as demonstrated in [27], $\omega_{m}$ and $\omega_{n}$ are not the real natural frequencies of the whole system. Neither is $\omega_{b}$ the real corner frequency. Here, these notations are employed just for dimensionless representations.

Replacing $s$ with $j \omega$ in (4), the frequency response functions in a dimensionless form can be obtained as

$$
H_{i}(j \lambda)=\frac{R_{n i}+j I_{n i}}{R_{m i}+j I_{m i}}, i=1, \ldots, 6,
$$

where $R_{n i}, I_{n i}, R_{m i}$, and $I_{m i}, i=1, \ldots, 6$ are functions with respect to $\lambda, \gamma, \delta$, and $\zeta$. The detailed representations are given in Appendix A.

\section{3. $H_{\infty}$ optimization for the IDVAs}

\subsection{Minmax optimization problem formulation}

The objective of the $H_{\infty}$ optimization is to minimize the maximum magnitude of the frequency response $\left|H_{i}(j \lambda)\right|, i=1, \ldots, 6$, which is known as the $H_{\infty}$ norm of $H_{i}(s)$ with 
Table 1: Admittance $Y(s)$ for each configuration in Fig. 2.

\begin{tabular}{cll}
\hline$Y_{1}(s)=b s+c$ & $Y_{2}(s)=\frac{1}{\frac{1}{b s}+\frac{1}{c}}$ & $Y_{3}(s)=\frac{1}{\frac{s}{k_{1}}+\frac{1}{c}+\frac{1}{b s}}$ \\
$Y_{4}(s)=\frac{1}{\frac{1}{\frac{k_{1}}{s}+c}+\frac{1}{b s}}$ & $Y_{5}(s)=\frac{1}{\frac{1}{\frac{k_{1}}{s}+b s}+\frac{1}{c}}$ & $Y_{6}(s)=\frac{1}{\frac{1}{b s+c}+\frac{s}{k_{1}}}$ \\
\hline
\end{tabular}

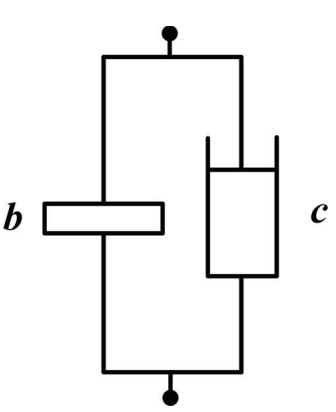

(a) $\mathrm{C} 1$

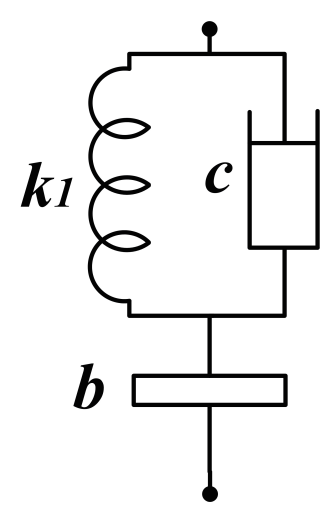

(d) $\mathrm{C} 4$

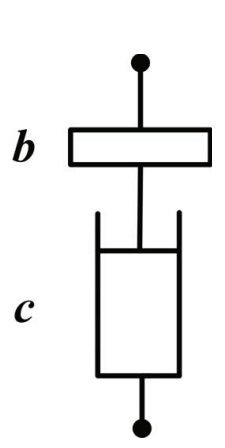

(b) $\mathrm{C} 2$

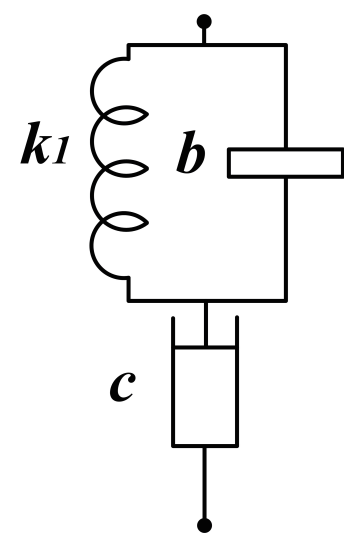

(e) $\mathrm{C} 5$

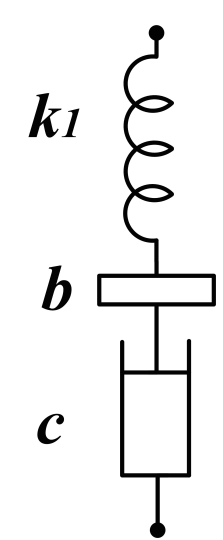

(c) $\mathrm{C} 3$

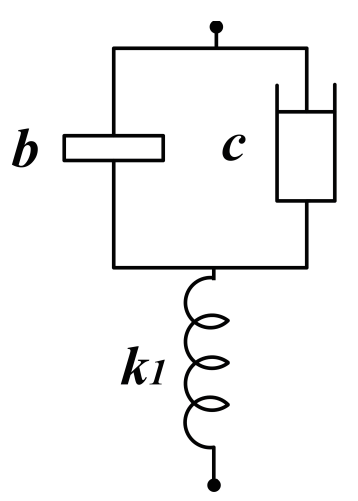

(f) $\mathrm{C} 6$

Figure 2: The employed inerter-based networks as $Y(s)$ in Fig. 1. 
$s=j \lambda$. For the TDVA, the fixed-point method [1] is commonly used to analytically obtain the optimal parameters [1, Chapter 3.3]. Since there always exist more than two fixed points with respect to the damping ratio for IDVAs, it is difficult to obtain simple and analytical representations for optimal parameters. Given this fact, in this paper, a minmax optimization problem is formulated as follows to directly minimize the magnitude at resonance frequencies.

For a given mass ratio $\mu$, solving the follow minmax problem

$$
\min _{\delta, \gamma, \eta, \zeta}\left(\max _{\lambda_{l}}\left(\left|H_{i}\left(j \lambda_{l}\right)\right|\right)\right), i=1, \ldots, 6
$$

subject to $\delta \geq 0, \gamma \geq 0, \eta \geq 0, \zeta \geq 0$, and $\lambda_{l}, l=1, \ldots, N$, are the real and positive solutions of the following equation

$$
\frac{\partial\left|H_{i}(j \lambda)\right|^{2}}{\partial \lambda^{2}}=0,
$$

where $i=1, \ldots, 6$ corresponds to the six IDVAs in Fig. 2, respectively.

The underlying idea of the minmax problem (8) and (9) is, instead of using the fixed points to approximately minimize the $H_{\infty}$ norm as done in the fixed-point method [1], here the resonance frequencies are directly used to exactly minimize the $H_{\infty}$ norm. This is inspired by the method in [4], where the two resonance frequencies were employed to derive the exact solutions for the TDVA. Note that the solution set of (9), that is $\lambda_{l}, l=1, \ldots, N$, contains the resonance frequencies, anti-resonance frequencies, and other frequencies where the curves horizontally pass through. Since the largest magnitude of the frequency response, representing the $H_{\infty}$ norm of the transfer function, only occurs at resonance frequencies, it is sufficient to minimize $\max _{\lambda_{l}}\left(\left|H_{i}\left(j \lambda_{l}\right)\right|\right), l=1, \ldots, N$, to obtain the optimal $H_{\infty}$ norm of the transfer function $H_{i}(s)$.

Equation (9) can be transformed into a polynomial function with respect to $\lambda^{2}$ as follows. From (7), $\left|H_{i}(j \lambda)\right|^{2}$ can be written as

$$
\left|H_{i}(j \lambda)\right|^{2}=\frac{n}{m},
$$

where $n=R_{n i}^{2}+I_{n i}^{2}, m=R_{m i}^{2}+I_{m i}^{2}$. Since

$$
\frac{\partial\left|H_{i}(j \lambda)\right|^{2}}{\partial \lambda^{2}}=\frac{n^{\prime} m-m^{\prime} n}{m^{2}},
$$

where $n^{\prime}=\frac{\partial n}{\partial \lambda^{2}}$ and $m^{\prime}=\frac{\partial m}{\partial \lambda^{2}},(9)$ is equivalent to

$$
n^{\prime} m-m^{\prime} n=0,
$$

which is an equation of $\lambda^{2}$ with different orders for different configurations.

Problem (8) and (10) is a constrained optimization problem, and the equality constraint (10) can be transformed into the objective function by employing $\lambda_{l}=f(\delta, \gamma, \eta, \zeta)$. In this paper, a direct search method is employed to solve the constrained optimization problem (8) and (10) by using the Matlab solver patternsearch with multiple starting points. 
3.2. Comparison between the TDVA and IDVAs

For the TDVA, the optimal parameters can be analytically obtained as [1]:

$$
\gamma_{o p t}=\sqrt{\frac{1}{1+\mu}}, \zeta_{o p t}=\sqrt{\frac{3 \mu}{8(1+\mu)}},
$$

and the optimal height at the two fixed points are $\sqrt{\frac{2+\mu}{\mu}}$.

\subsubsection{Performance limitation of $C 1$ and $C 2$}

In this subsection, it will be demonstrated that configurations $C 1$ and $C 2$ provide no improvement for the $H_{\infty}$ performance compared with the TDVA.

For configuration $C 1$, by directly using the fixed-point method in [1], the optimal parameters for $C 1$ can be analytically obtained as

$$
\gamma_{o p t}=\frac{\sqrt{1+(1+\mu) \delta}}{1+\mu}, \quad \zeta_{o p t}=\sqrt{\frac{3 \mu}{8(1+\mu)}},
$$

and the optimal height at the two fixed points are $\sqrt{\frac{2+\mu+2 \delta(1+\mu)}{\mu}}$. It is obvious that the optimal $\delta$ is 0 , which means that the parallel inerter in configuration $C 1$ provides no improvement in the $H_{\infty}$ optimization. Such an observation is shown in Fig. 3 with $\mu=0.1$.

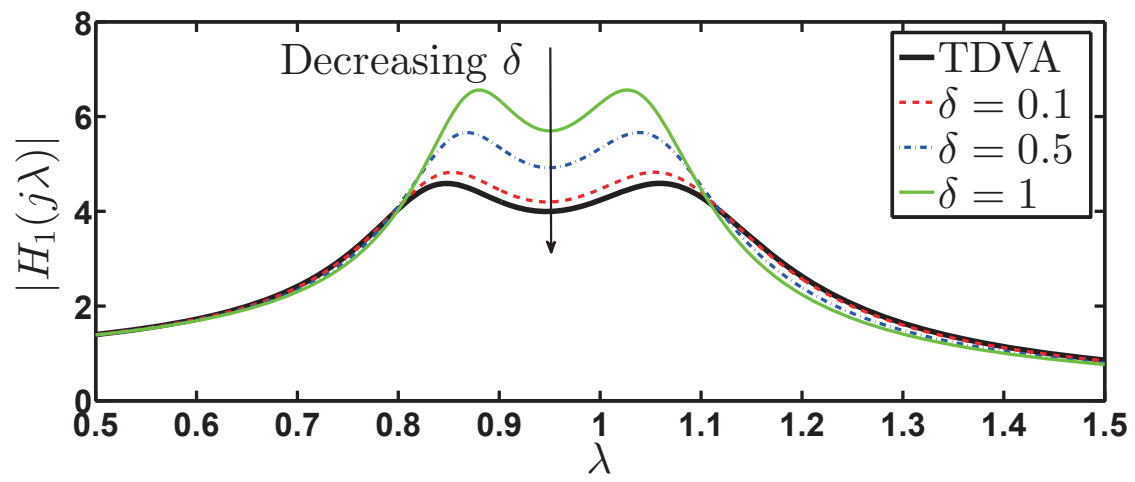

Figure 3: Comparison between the TDVA and $C 1$ when $\mu=0.1$ with different $\delta$.

The minmax optimization method proposed in this paper is also applicable for $C 1$ and a comparison between the method in this paper and the fixed-point method is shown in Fig. 4. As shown in Fig. 4, the results by these two methods highly coincide with each other and the results are consistent with the analytical solutions in [4, Table 2], which demonstrates the effectiveness of the method in this paper.

In what follows, it will be shown that for configuration $C 2$, the series-connected inerter provides no improvement for the $H_{\infty}$ performance as well. To show the influence of $\delta$, the problem (8) is slightly modified as: for a given $\mu$ and $\delta$,

$$
\min _{\gamma, \zeta}\left(\max _{\lambda_{l}}\left(\left|H_{2}\left(j \lambda_{l}\right)\right|\right)\right)
$$




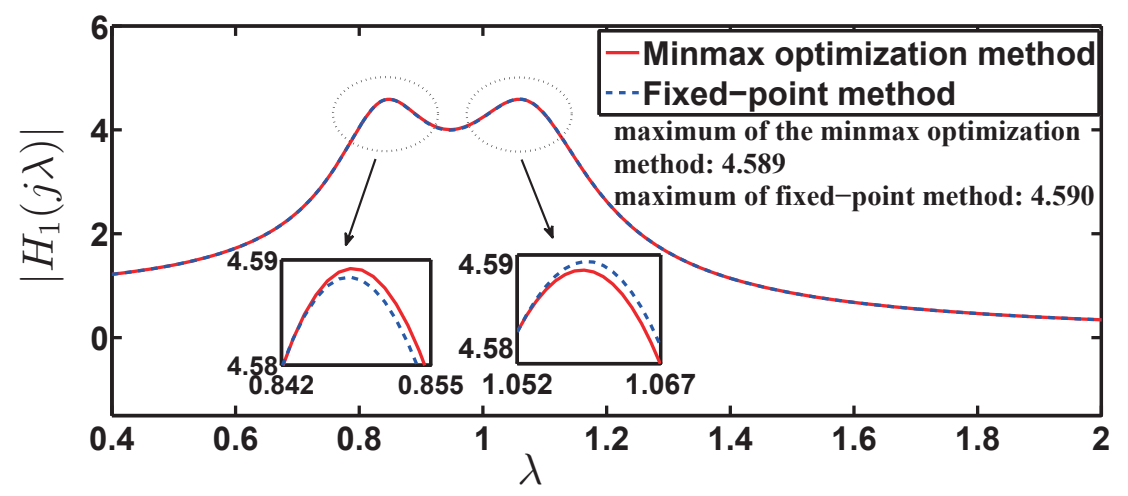

Figure 4: Comparison between the minmax optimization method in this paper and the fixed-point method when $\mu=0.1$.

subject to $\gamma \geq 0, \eta \geq 0, \zeta \geq 0$, and $\lambda_{l}, l=1, \ldots, N$, are the real and positive solutions of (10). Fig. 5 shows the comparison between $C 2$ with different $\delta$ and the TDVA when $\mu=0.1$, where it is clearly shown that the maximum of $\left|H_{2}(j \lambda)\right|$ is decreased by increasing $\delta$ and if $\delta$ is sufficiently large, the frequency response of $C 2$ coincides with that of the TDVA. Such an observation is also confirmed by other choices of $\mu$, as shown in Fig. 6. Therefore, it is sufficient to conclude that for a single series arrangement of an inerter and a damper, the series inerter provides no improvement for the $H_{\infty}$ performance of the isolation system.

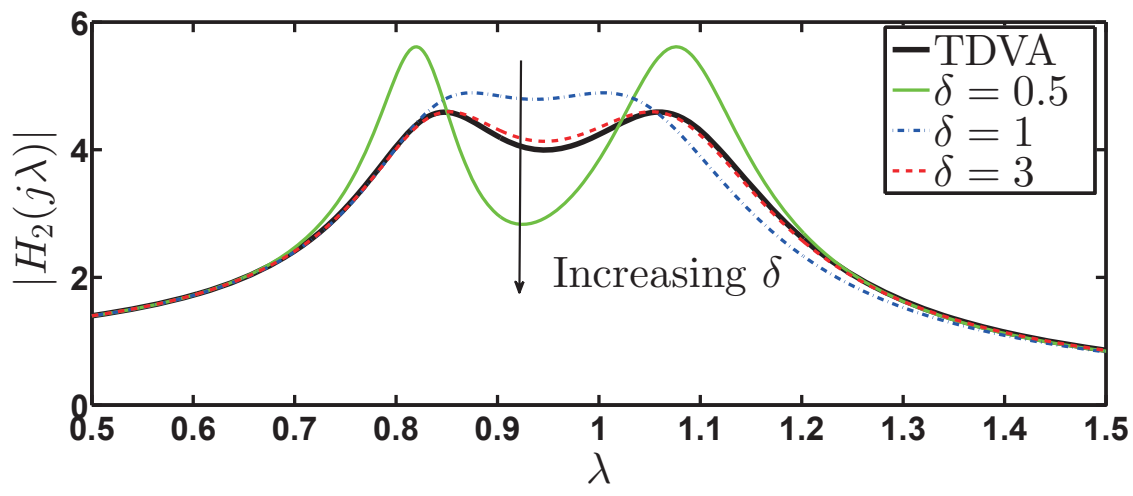

Figure 5: Comparison between the TDVA and $C 2$ when $\mu=0.1$ with different $\delta$.

The IDVAs $C 1$ and $C 2$ represent the two ways of adding an inerter to the TDVA, that is, the parallel connection $(C 1)$ and the series connection $(C 2)$. Now, it has been demonstrated that adding a single inerter alone to the TDVA, no matter it is in parallel connection or in series connection, provides no improvement for the $H_{\infty}$ performance. Therefore, other degrees of freedom should be introduced, which is the motivation of introducing IDVAs $C 3$, $C 4, C 5$, and $C 6$ by adding an inerter together with a spring to the TDVA.

\subsubsection{Performance benefits of $C 3, C 4, C 5$, and $C 6$}

In this subsection, it will be shown that after adding another degree of freedom, that is the spring $k_{1}$, the $H_{\infty}$ performance will be significantly improved compared with the TDVA.

The optimization problem (8) with the constraint (10) is solved for configurations $C 3$, $C 4, C 5$ and $C 6$, separately, where a 9th-order polynomial of equation (10) with respect to 


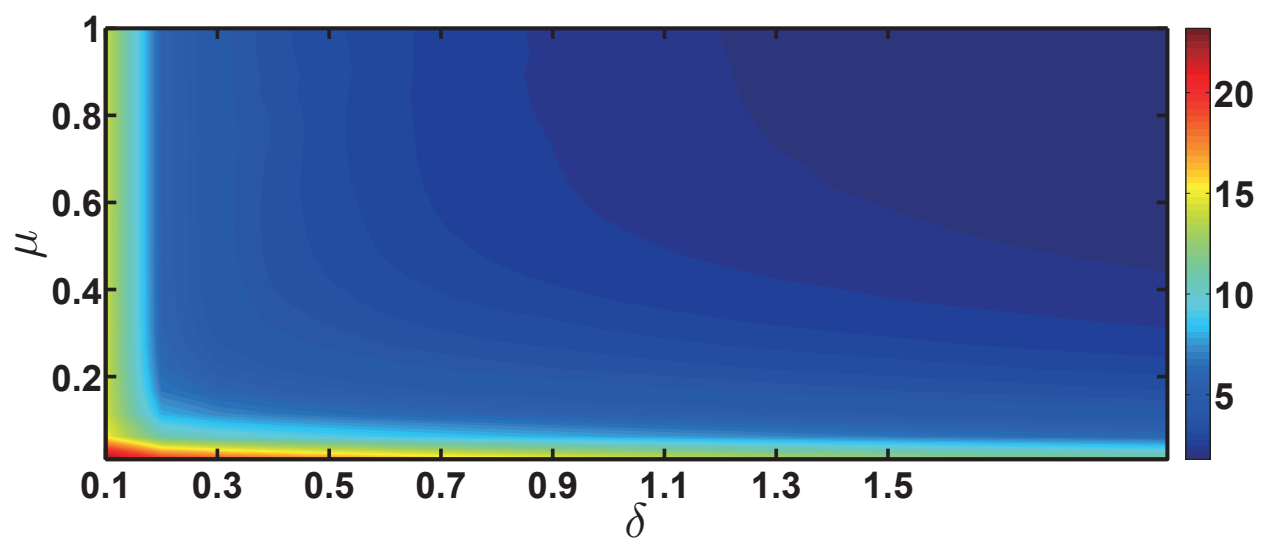

Figure 6: $\max \left(\left|H_{2}(j \lambda)\right|\right)$ with different $\mu$ and $\delta$.

$\lambda^{2}$ is obtained. The exact solutions of the TDVA in [4] are employed for comparison and the detailed parameter values are shown in Tables 2, 3, and 4. Table 2 shows that all the IDVAs $C 3, C 4, C 5$ and $C 6$ can improve the $H_{\infty}$ performance compared with the TDVA, where $C 3$ performs the best and the order of the performance is $C 3>C 6>C 4>C 5>T D V A$ (">" means performing better) with an exception for $\mu>=1$. However, since the mass ratio is normally quite small and practically less than $0.25[42,43]$, it is sufficient to conclude that $C 3>C 6>C 4>C 5>T D V A$. Such an conclusion is also confirmed by Fig. 7, where the comparison of the IDVAs over the TDVA in the range of $0<\mu \leq 0.25$ is shown. As shown in the right figure of Fig. 7, $8 \%$ to $26 \%$ improvement can be obtained for the IDVAs. The other parameters in the range of $0<\mu \leq 0.25$ are depicted in Fig. 8. It should be noted that although the optimal $\gamma$ and $\zeta$ for $C 3$ are almost identical to the TDVA, as shown in Table 3 and Fig. 8, over $22 \%$ improvement can be provided by $C 3$ compared with the TDVA. Moreover, the spring $k_{1}$ is better to be in series connection for the $H_{\infty}$ performance, given the fact that $C 3$ and $C 6$ are superior to $C 4$ and $C 5$.

The frequency responses of the IDVAs and the TDVA when $\mu=0.1$ are shown in Fig. 9, where one sees that the magnitudes of the IDVAs around 1 are much flatter than those of the TDVA, and the effective frequency band is much larger than that of the TDVA.

Table 2: maximum magnitude $\max |H(j \lambda)|$ in the $H_{\infty}$ optimization.

\begin{tabular}{llllll}
\hline$\mu$ & TDVA $[4]$ & $C 3$ & $C 4$ & $C 5$ & $C 6$ \\
\hline 0.01 & 14.1796 & 11.0330 & 11.0860 & 12.9216 & 11.0351 \\
0.02 & 10.0530 & 7.8340 & 7.9064 & 9.1498 & 7.8352 \\
0.05 & 6.4080 & 5.0159 & 5.1194 & 5.8051 & 5.0210 \\
0.1 & 4.5892 & 3.6175 & 3.7448 & 4.1379 & 3.6208 \\
0.2 & 3.3254 & 2.6552 & 2.7986 & 2.9877 & 2.6616 \\
0.5 & 2.2480 & 1.8513 & 1.9941 & 2.0198 & 1.8521 \\
1 & 1.7457 & 1.4893 & 1.6127 & 1.5809 & 1.4893 \\
2 & 1.4279 & 1.2697 & 1.3629 & 1.3157 & 1.2697 \\
5 & 1.1942 & 1.1166 & 1.1702 & 1.1766 & 1.1166 \\
10 & 1.1033 & 1.0602 & 1.0918 & 1.0934 & 1.0603 \\
\hline
\end{tabular}


Table 3: Optimal natural frequency ratio $\gamma$ and damping ratio $\zeta$ in the $H_{\infty}$ optimization.

(a) Optimal natural frequency ratio $\gamma$

\begin{tabular}{llllll}
\hline$\mu$ & TDVA $[4]$ & $C 3$ & $C 4$ & $C 5$ & $C 6$ \\
\hline 0.01 & 0.9902 & 0.9900 & 0.9957 & 0.9712 & 0.9842 \\
0.02 & 0.9802 & 0.9802 & 0.9911 & 0.9493 & 0.9684 \\
0.05 & 0.9520 & 0.9520 & 0.9766 & 0.9090 & 0.9242 \\
0.1 & 0.9083 & 0.9083 & 0.9499 & 0.8501 & 0.8642 \\
0.2 & 0.8319 & 0.8319 & 0.8931 & 0.7538 & 0.7693 \\
0.5 & 0.6642 & 0.6643 & 0.7514 & 0.5681 & 0.5604 \\
1 & 0.4973 & 0.4971 & 0.5882 & 0.4041 & 0.3979 \\
2 & 0.3307 & 0.3302 & 0.4100 & 0.2547 & 0.2526 \\
5 & 0.1646 & 0.1641 & 0.2145 & 0.2004 & 0.1197 \\
10 & 0.0889 & 0.0893 & 0.1198 & 0.1118 & 0.0652 \\
\hline & & $(\mathrm{b})$ Optimal damping ratio $\zeta$ & \\
\hline$\mu$ & TDVA $[4]$ & $C 3$ & $C 4$ & $C 5$ & $C 6$ \\
\hline 0.01 & 0.0603 & 0.0547 & 0.0025 & 0.0655 & 0.0025 \\
0.02 & 0.0841 & 0.0769 & 0.0065 & 0.0973 & 0.0073 \\
0.05 & 0.1276 & 0.1199 & 0.0224 & 0.1477 & 0.0270 \\
0.1 & 0.1686 & 0.1657 & 0.0505 & 0.2086 & 0.0593 \\
0.2 & 0.2101 & 0.2244 & 0.0981 & 0.2919 & 0.1180 \\
0.5 & 0.2402 & 0.3175 & 0.2012 & 0.4294 & 0.3047 \\
1 & 0.2235 & 0.3894 & 0.2905 & 0.5359 & 0.4354 \\
2 & 0.1749 & 0.4505 & 0.3779 & 0.6325 & 0.5498 \\
5 & 0.1002 & 0.5057 & 0.4525 & 0.5163 & 0.6593 \\
10 & 0.0581 & 0.5288 & 0.4804 & 0.5313 & 0.6841 \\
\hline & & & & &
\end{tabular}
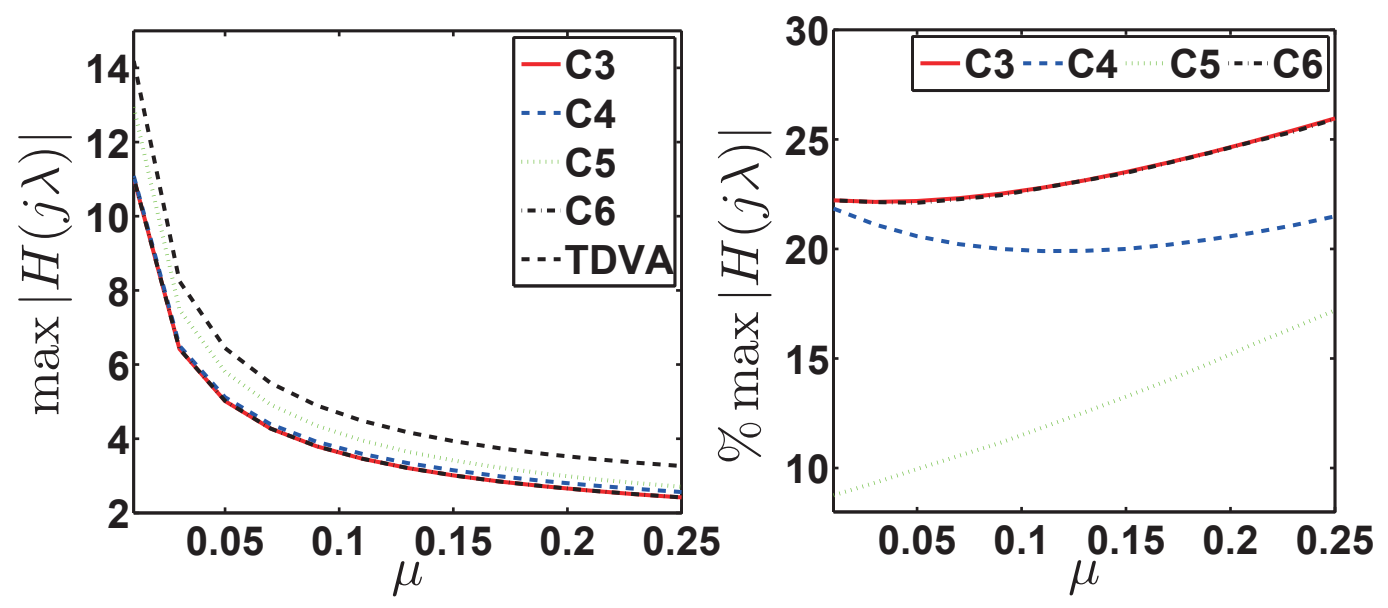

Figure 7: Maximum magnitude comparison between the IDVAs and the TDVA (left figure) and percentage improvement of the IDVAs with respect to the TDVA (right figure). 
Table 4: Optimal inertance-to-mass ratio $\delta$ and corner frequency ratio $\eta$ in the $H_{\infty}$ optimization.

(a) Optimal inertance-to-mass ratio $\delta$

\begin{tabular}{lllll}
\hline$\mu$ & $C 3$ & $C 4$ & $C 5$ & $C 6$ \\
\hline 0.01 & 0.0238 & 0.0234 & 2.2791 & 0.0228 \\
0.02 & 0.0473 & 0.0453 & 1.8105 & 0.0448 \\
0.05 & 0.1156 & 0.1069 & 1.6782 & 0.0989 \\
0.1 & 0.2208 & 0.1930 & 1.5320 & 0.1538 \\
0.2 & 0.4082 & 0.3212 & 1.1521 & 0.2126 \\
0.5 & 0.8256 & 0.5719 & 0.6919 & 0.2426 \\
1 & 1.2552 & 0.7785 & 0.3130 & 0.2009 \\
2 & 1.7228 & 0.9703 & 0.1423 & 0.1364 \\
5 & 2.2540 & 1.1307 & 3.9018 & 0.0627 \\
10 & 2.4989 & 1.2089 & 3.6257 & 0.0339 \\
\hline & $(\mathrm{b})$ Optimal corner frequency ratio $\eta$ & \\
\hline$\mu$ & $C 3$ & $C 4$ & $C 5$ & $C 6$ \\
\hline 0.01 & 1.0051 & 0.9864 & 1.1242 & 1.0248 \\
0.02 & 1.0098 & 0.9745 & 1.1982 & 1.0492 \\
0.05 & 1.0248 & 0.9420 & 1.3341 & 1.1288 \\
0.1 & 1.0485 & 0.9013 & 1.5181 & 1.2454 \\
0.2 & 1.0940 & 0.8563 & 1.8754 & 1.4560 \\
0.5 & 1.2219 & 0.7713 & 2.8856 & 2.2775 \\
1 & 1.4061 & 0.7163 & 4.9686 & 3.5386 \\
2 & 1.7178 & 0.6629 & 9.6074 & 6.0835 \\
5 & 2.4169 & 0.6141 & 0.5009 & 14.5775 \\
10 & 3.2632 & 0.5780 & 0.4739 & 27.6261 \\
\hline & & & &
\end{tabular}
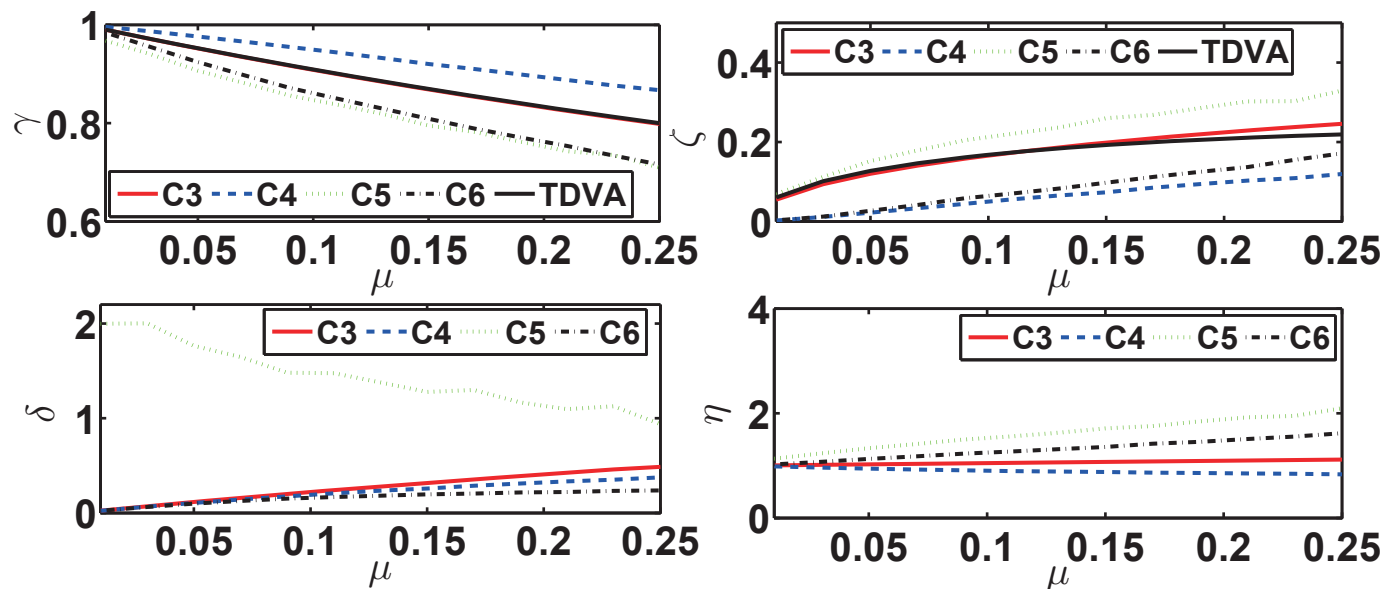

Figure 8: Optimal parameters in the $H_{\infty}$ optimization: natural frequency ratio $\gamma$ (up left); damping ratio $\zeta$ (up right); inertance-to-mass ratio $\delta$ (bottom left); corner frequency ratio $\eta$ (bottom right). 


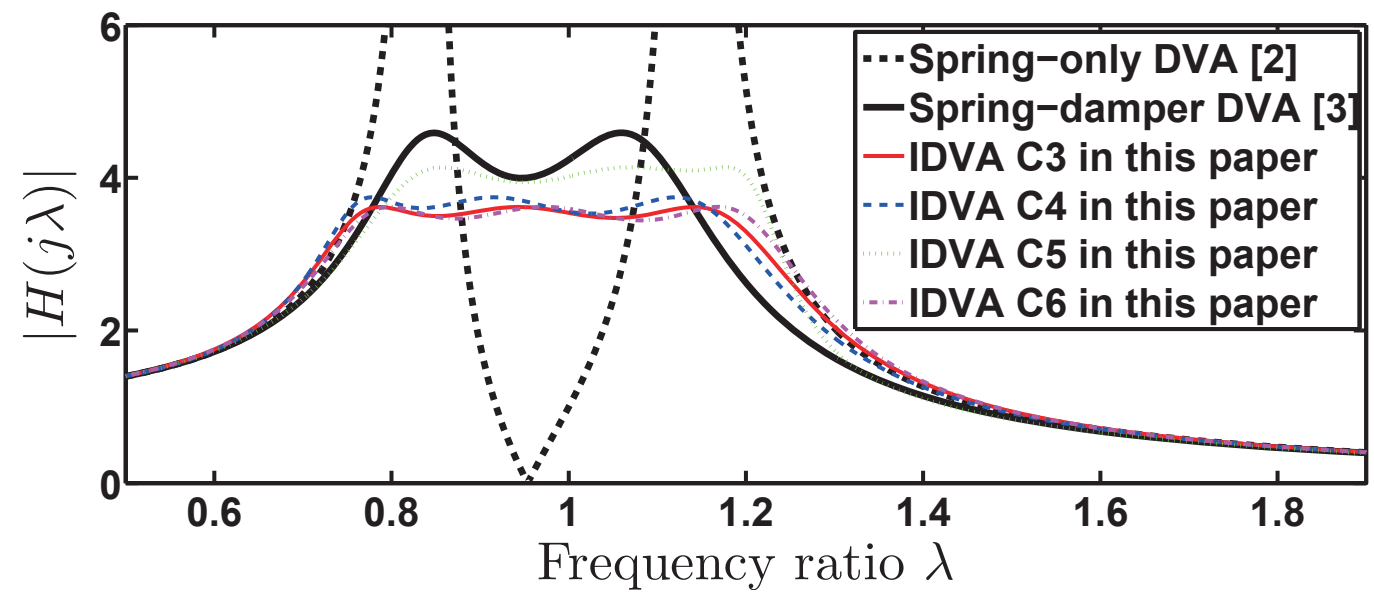

Figure 9: Comparison between the IDVAs and TDVA when $\mu=0.1$. The spring-only DVA is the first DVA proposed by Frahm in 1909 [2]. The spring-damper DVA is the TDVA proposed by Ormondroyd and Den Hartog in 1928 [3].

\section{4. $H_{2}$ optimization for the IDVAs}

\section{1. $\mathrm{H}_{2}$ performance measure and its analytical solution}

If the system is subjected to random excitation instead of sinusoidal excitation, the $\mathrm{H}_{2}$ optimization would be more desirable than the $H_{\infty}$ optimization $[9,39,40]$. The performance measure in the $H_{2}$ optimization is defined as $[9,39,40]$

$$
I=\frac{E\left[x^{2}\right]}{2 \pi S_{0} \omega_{n}},
$$

where $S_{0}$ is the uniform power spectrum density function. The mean square value of $x$ of the object mass $m$ can be calculated as

$$
E\left[x^{2}\right]=S_{0} \int_{-\infty}^{\infty}|H(j \lambda)|^{2} d_{\omega}=S_{0} \omega_{n} \int_{-\infty}^{\infty}|H(j \lambda)|^{2} d_{\lambda},
$$

where $H(j \lambda)$ is given in (7). Substituting (12) into (11), one obtains

$$
I=\frac{1}{2 \pi} \int_{-\infty}^{\infty}|H(j \lambda)|^{2} d_{\lambda},
$$

which is exactly the definition of the $H_{2}$ norm of the transfer function $\hat{H}(s)$ by replacing $j \lambda$ in $H(j \lambda)$ with the Laplace variable $s$.

Therefore, the $\mathrm{H}_{2}$ performance measure is rewritten as

$$
I=\|\hat{H}(s)\|_{2}^{2} .
$$

The analytical approach provided in [41, Chapter 2.6] will be employed to derive analytical solutions for IDVAs in the $\mathrm{H}_{2}$ optimization, which is briefly presented as follows.

For a stable transfer function $\hat{H}(s)$, its $H_{2}$ norm can be calculated as [41, Chapter 2.6]

$$
\|\hat{H}(s)\|_{2}^{2}=\left\|C(s I-A)^{-1} B\right\|_{2}^{2}=C L C^{T},
$$


where $A, B, C$ are the minimal state-space realization $\hat{H}(s)=C(s I-A)^{-1} B$ and $L$ is the unique solution of the Lyapunov equation

$$
A L+L A^{T}+B B^{T}=0 .
$$

We can write $\hat{H}(s)$

$$
\hat{H}(s)=\frac{b_{n-1} s^{n-1}+\ldots+b_{1} s+b_{0}}{s^{n}+a_{n-1} s^{n-1}+\ldots+a_{1} s+a_{0}}
$$

in its controllable canonical form below

$$
\dot{x}=A x+B u, y=C x,
$$

198 where

$$
A=\left[\begin{array}{ccccc}
0 & 1 & 0 & \ldots & 0 \\
0 & 0 & 1 & \ldots & 0 \\
\vdots & \vdots & \vdots & \vdots & \vdots \\
0 & 0 & 0 & \ldots & 1 \\
-a_{0} & -a_{1} & -a_{2} & \ldots & -a_{n-1}
\end{array}\right], B=\left[\begin{array}{c}
0 \\
0 \\
\vdots \\
0 \\
1
\end{array}\right], C=\left[b_{0}, b_{1}, b_{2} \ldots b_{n-1}\right]
$$

\subsection{Comparison between the TDVA and IDVAs}

For the TDVA, the $H_{2}$ performance measure can be obtained as

$$
I_{T D V A}=\frac{\gamma(1+\mu) \zeta}{\mu}+\frac{1-(\mu+2) \gamma^{2}+(1+\mu)^{2} \gamma^{4}}{4 \mu \gamma \zeta},
$$

and the optimal $\gamma$ and $\zeta$ are

$$
\begin{aligned}
\gamma_{T D V A, o p t} & =\sqrt{\frac{\mu+2}{2(1+\mu)^{2}}}, \\
\zeta_{T D V A, o p t} & =\sqrt{\frac{(3 \mu+4) \mu}{8(\mu+1)(\mu+2)}} .
\end{aligned}
$$

Substituting $\gamma_{T D V A, o p t}$ and $\zeta_{T D V A, o p t}$ into (16), one obtains the optimal $I_{T D V A, o p t}$ as

$$
I_{T D V A, o p t}=\sqrt{\frac{3 \mu+4}{4(\mu+1) \mu}} .
$$

4.2.1. Performance limitation of $C 1$ and $C 2$

The $H_{2}$ performance measures for $C 1$ and $C 2$ can be obtained as

$$
\begin{aligned}
I_{C 1} & =\frac{\gamma(1+\mu) \zeta}{\mu}+\frac{1}{4 \mu \gamma \zeta}\left(\delta^{2}-2\left((1+\mu) \gamma^{2}-1\right) \delta+1-(\mu+2) \gamma^{2}+(1+\mu)^{2} \gamma^{4}\right) \\
& =I_{T D V A}+\frac{1}{4 \mu \gamma \zeta}\left(\delta^{2}+a_{C 1,1} \delta\right) \\
I_{C 2} & =\left(a_{C 2,2} \delta^{-2}+a_{C 2,1} \delta^{-1}+a_{C 2,0}\right) \zeta+\frac{1-(\mu+2) \gamma^{2}+(1+\mu)^{2} \gamma^{4}}{4 \mu \gamma \zeta} \\
& =I_{T D V A}+\left(a_{C 2,2} \delta^{-2}+a_{C 2,1} \delta^{-1}\right) \zeta
\end{aligned}
$$


where

$$
\begin{aligned}
a_{C 1,1} & =-2\left((1+\mu) \gamma^{2}-1\right), \\
a_{C 2,2} & =\frac{\gamma}{\mu}\left((1+\mu)^{3} \gamma^{4}-2(1+\mu) \gamma^{2}+1\right), \\
a_{C 2,1} & =\frac{\gamma}{\mu}\left(2+\mu-2(1+\mu)^{2} \gamma^{2}\right), \\
a_{C 2,0} & =\frac{\gamma(1+\mu)}{\mu} .
\end{aligned}
$$

The following proposition can be obtained.

Proposition 1. For the $\mathrm{H}_{2}$ performance, $C 1$ performs no better than the TDVA.

Proof. See Appendix B.

Proposition 2. For the $\mathrm{H}_{2}$ performance, C2 performs slightly better than the TDVA, but only at most $0.32 \%$ improvement can be achieved when $\mu \leq 1$.

Proof. See Appendix C.

Now, we have demonstrated that for the $H_{2}$ performance, $C 1$ performs no better than the TDVA and $C 2$ provides negligible improvement over the TDVA. This means that adding an inerter alone to the TDVA provides limited improvement for the $H_{2}$ performance, and therefore, another four IDVAs $C 3, C 4, C 5$, and $C 6$ are proposed by adding an inerter together with a spring to the TDVA. It will be shown in the following sections that in this way, the $\mathrm{H}_{2}$ performance can be significantly improved.

\subsubsection{Performance benefits of $C 3, C 4, C 5$, and $C 6$}

In this subsection, it will be analytically demonstrated that for the $H_{2}$ performance, IDVAs $C 3, C 4, C 5$, and $C 6$ perform surely better than the TDVA, and an optimization problem will be formulated to find the optimal parameters.

By using the method shown in Subsection 4.1, the analytical representations of the $\mathrm{H}_{2}$ performance measures for $C 3, C 4, C 5$, and $C 6$ are calculated and the detailed equations are shown in Appendix D. Denote the optimal $H_{2}$ performances of $C 3, C 4, C 5$, and $C 6$ as $I_{C 3, \text { opt }}, I_{C 4, \text { opt }}, I_{C 5, \text { opt }}, I_{C 6, \text { opt }}$, respectively. The following proposition can be obtained.

Proposition 3. For the $\mathrm{H}_{2}$ performance, IDVAs $C 3$ and $C 5$ always perform better than the TDVA, that is, the following inequalities hold:

$$
\begin{aligned}
I_{C 3, \mathrm{opt}} & <I_{T D V A, o p t}, \\
I_{C 5, \mathrm{opt}} & <I_{T D V A, o p t},
\end{aligned}
$$

and if $\mu \leq 1, I D V A s C 4$ and $C 6$ always perform better than the TDVA, that is, the following inequalities hold:

$$
\begin{aligned}
& I_{C 4, \text { opt }}<I_{T D V A, o p t}, \\
& I_{C 6, \text { opt }}<I_{T D V A, o p t},
\end{aligned}
$$

where $I_{T D V A, o p t}$ is the optimal $H_{2}$ performance for the TDVA given by (19). 
Proof. See Appendix E.

Remark 3. The condition $\mu \leq 1$ for $C 4$ and $C 6$ in Proposition 3 is only a sufficient condition, which means that for the case $\mu>1$, it is also possible that the inequalities (26) and (27) hold. However, such a condition introduces no conservativeness for DVA applications, as the mass ratio $\mu$ is normally less than 1 in practice (typically less than 0.25) [42, 43].

Since the IDVAs C3, C4, C5, C6 can always reduce to the TDVA by setting the spring stiffness $k_{1}$ (or $\eta$ ) and inertance $b$ (or $\delta$ ) to 0 or $\infty$, the conclusions $I_{C i, \text { opt }} \leq I_{T D V A, o p t}$, $i=3,4,5,6$ always hold. However, Proposition 3 demonstrates the existence of finite $\eta$ and $\delta$ such that the IDVAs $C 3, C 4, C 5$, and $C 6$ are surely better than the TDVA.

To determine the optimal values of $\delta, \gamma, \eta$, and $\zeta$, the following optimization problem should be solved.

$$
\min _{\delta, \gamma, \eta, \zeta} I_{C i}, i=3,4,5,6,
$$

subject to $\delta>0, \gamma>0, \eta>0$, and $\zeta>0$.

Analytical solutions of $C 3$ : Problem (28) can be analytically solved for $C 3$, where the optimal parameters for $C 3$ are obtained as follows

$$
\begin{aligned}
\gamma_{C 3, \text { opt }} & =\sqrt{\frac{\sqrt{17 \mu^{2}+32 \mu+16}-\mu}{4(1+\mu)^{2}}}, \\
\eta_{C 3, \text { opt }} & =\sqrt{\frac{1-2(1+\mu) \gamma_{C 3, o p t}^{2}+(1+\mu) \gamma_{C 3, \text { opt }}^{4}}{\left(1-(2+3 \mu) \gamma_{C 3, \text { opt }}^{2}+(1+\mu)^{2} \gamma_{C 3, o p t} 64\right) \gamma_{C 3, o p t}^{2}}}, \\
\delta_{C 3, \text { opt }} & =-\frac{2 \hat{a}_{C 3,2}}{\hat{a}_{C 3,1}}, \\
\zeta_{C 3, \text { opt }} & =\sqrt{\frac{1-(\mu+2) \gamma_{C 3, o p t}^{2}+(1+\mu)^{2} \gamma_{C 3, o p t}^{4}}{4 \mu \gamma_{C 3, o p t}\left(\hat{a}_{C 3,2} \delta_{C 3, o p t}^{2}+\hat{a}_{C 3,1} \delta_{C 3, o p t}^{-1}+\hat{a}_{C 3,0}\right)}},
\end{aligned}
$$

where $\hat{a}_{C 3,2}, \hat{a}_{C 3,1}$, and $\hat{a}_{C 3,0}$ are obtained by setting $\gamma=\gamma_{C 3, o p t}$ and $\eta=\eta_{C 3, o p t}$ for $a_{C 3,2}$, $a_{C 3,1}$, and $a_{C 3,0}$, respectively. For the representations of $a_{C 3,2}, a_{C 3,1}$, and $a_{C 3,0}$, see Appendix D.

The analytical solutions $\delta, \gamma$, and $\eta$ are derived by successively setting the first derivatives of $I_{C 3}$ with respect to $\delta, \eta$, and $\gamma$ as 0 , and then checking the sign of the second derivatives at stationary points. The optimal $\zeta_{C 3, o p t}$ is derived due to the fact that both parts on the right hand side of (D.1) of $I_{C 3}$ are positive.

Solutions of $C 4, C 5$, and $C 6$ : The analytical solutions of $C 4, C 5$, and $C 6$ cannot be obtained due to the high order equations (more than 4th-order) involved in the derivation. However, the optimal solutions of $\eta$ and $\zeta$ can be analytically represented with respect to $\delta$ 


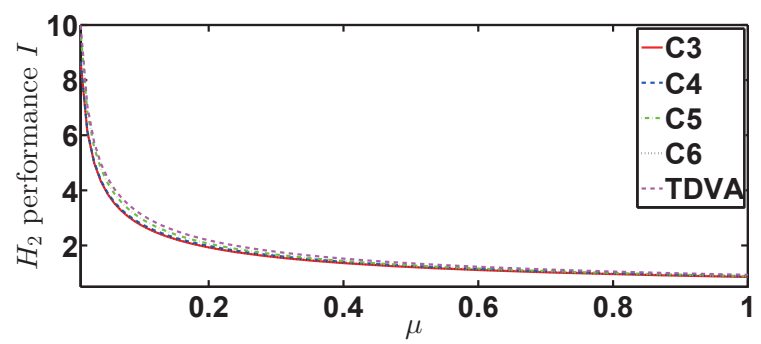

(a)

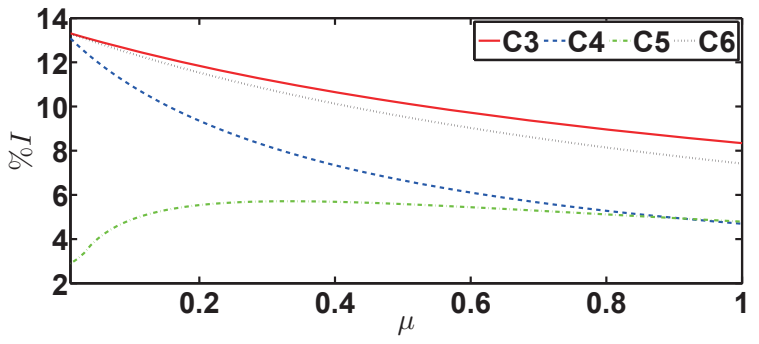

(b)

Figure 10: Comparison between IDVAs and the TDVA. (a) the $H_{2}$ performance; (b) Percentage improvement of IDVAs with respect to the TDVA.

and $\gamma$ as follows:

$$
\begin{aligned}
\eta_{C 4, \text { opt }} & =\frac{\sqrt{-\left(g_{C 4,1} \delta+f_{C 4,1}\right)\left(2 f_{C 4,2}+2 g_{C 4,2} \delta+2 l_{C 4,2} \delta^{2}\right)}}{2\left(f_{C 4,2}+g_{C 4,2} \delta+l_{C 4,2} \delta^{2}\right)}, \\
\zeta_{C 4, \text { opt }} & =\sqrt{\frac{l_{C 4,2} \eta^{4} \delta^{2}+l_{C 4,1} \delta+l_{C 4,0}}{a_{C 4,2} \delta^{-2}+a_{C 4,1} \delta^{-1}+a_{C 4,0}}}, \\
\delta_{C 5, \text { opt }} & =-\frac{2 a_{C 5,2}}{a_{C 5,1}}, \\
\zeta_{C 5, \text { opt }} & =\sqrt{\frac{1-(\mu+2) \gamma^{2}+(1+\mu)^{2} \gamma^{4}}{4 \mu \gamma\left(a_{C 5,2} \delta_{C 5, o p t}^{-2}+a_{C 5,1} \delta_{C 5, o p t}^{-1}+a_{C 5,0}\right)}}, \\
\zeta_{C 6, \text { opt }} & =\sqrt{\frac{l_{C 6,2} \eta^{4} \delta^{2}+l_{C 6,1} \delta+l_{C 6,0}}{a_{C 6,2} \delta^{-2}+a_{C 6,1} \delta^{-1}+a_{C 6,0}}} .
\end{aligned}
$$

Correspondingly substituting the optimal representations above into $I_{C i}, i=4,5,6$, the problem (28) for $C i, i=4,5,6$ reduces to a nonlinear programming problem with two unknown variables $\delta$ and $\gamma$ for $C 4$ and $C 5$, and with three unknown variables $\delta, \gamma$ and $\eta$ for $C 6$, which can be efficiently solved by using the Matlab solver fmincon and GlobalSearch in Global Optimization Toolbox.

Fig. 10 and Fig. 11 depict the comparison between IDVAs $C 3, C 4, C 5, C 6$ and the TDVA when $0 \leq \mu \leq 1$. As shown in Fig. 10(b), $C 3$ performs the best, and more than $10 \%$ improvement with respect to the TDVA can be obtained by $C 3, C 4$ and $C 6$. Similar to the $H_{\infty}$ performance, the spring $k_{1}$ is better to be in series connection for the $H_{2}$ performance, given the fact that $C 3$ and $C 6$ are superior to $C 4$ and $C 5$.

\section{Conclusions}

In this paper, the performance of inerter-based dynamic vibration absorbers (IDVAs) has been investigated, where the proposed IDVAs were a parallel arrangement of a spring and an inerter-based mechanical network. Both $H_{\infty}$ and $H_{2}$ performances were considered. The $H_{\infty}$ performance optimization problem was formulated in a minmax framework and solved by using a direct search optimization method; while in the $\mathrm{H}_{2}$ optimization, an analytical method 


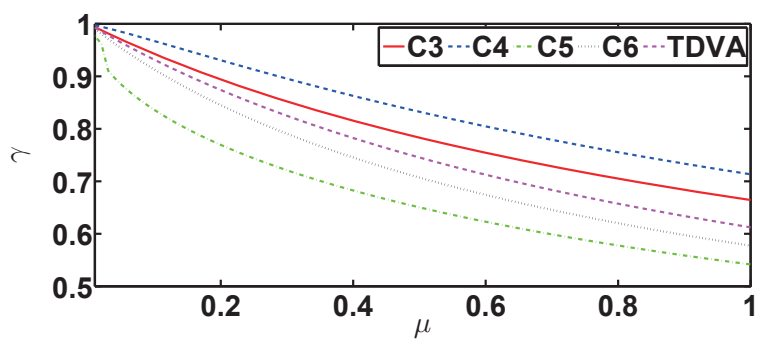

(a)

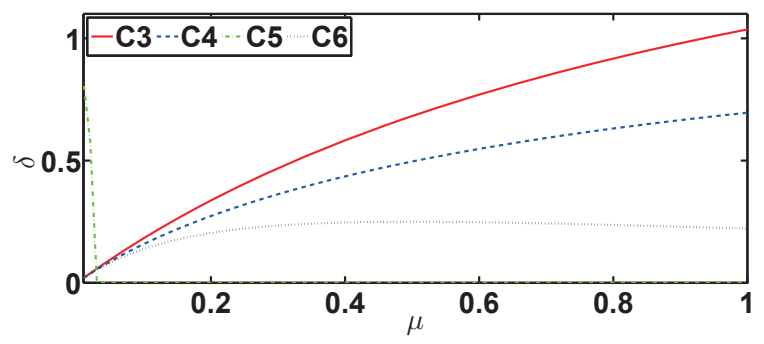

(c)

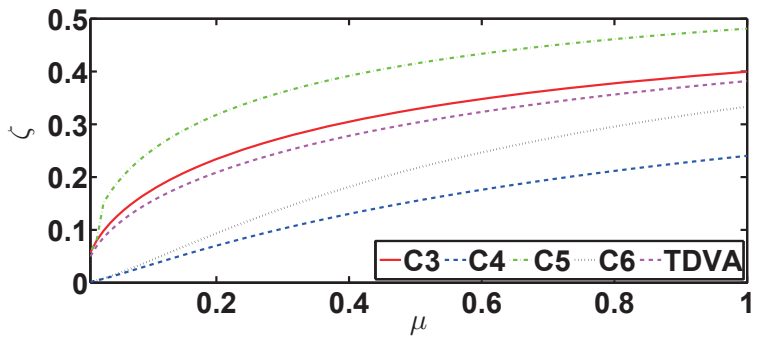

(b)

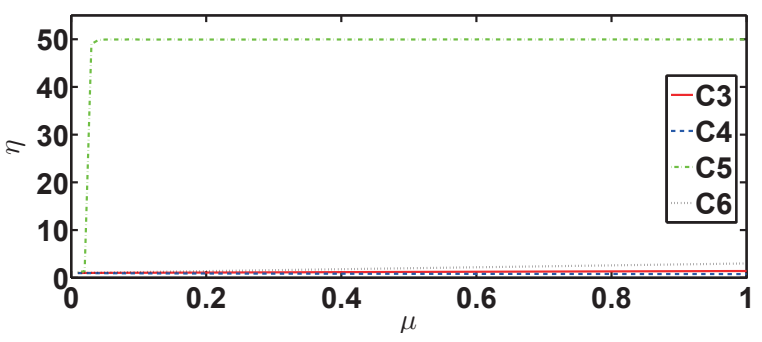

(d)

Figure 11: Optimal parameters: (a) optimal $\gamma$; (b) optimal $\zeta$; (c) optimal $\delta$; (d) optimal $\eta$.

was employed to calculate the $H_{2}$ performance measures. Comparisons between the proposed IDVAs and the traditional dynamic vibration absorber (TDVA) were conducted. The results showed that adding one inerter alone to the TDVA, no matter it is in parallel connection $(C 1)$ or in series connection $(C 2)$, provided no improvement for the $H_{\infty}$ performance, and negligible improvement (less than $0.32 \%$ improvement over the TDVA when the mass ratio less than 1) for the $\mathrm{H}_{2}$ performance. This demonstrated the necessity of introducing another degree of freedom together with the inerter to the TDVA, and then the IDVAs $C 3, C 4, C 5$, and $C 6$ were proposed by adding an inerter together with a spring to the TDVA. Significant improvement was obtained by IDVAs $C 3, C 4, C 5$, and $C 6$. For the $H_{\infty}$ performance, numerical simulations showed that over $20 \%$ improvement was achieved compared with the TDVA and the effective frequency band can be enlarged by using inerter; while for the $H_{2}$ performance, it was analytically demonstrated that IDVAs $C 3, C 4, C 5$, and $C 6$ were surely better than the TDVA by carefully choosing the parameters, and over $10 \%$ improvement was obtained in the numerical simulation. 


$$
\begin{aligned}
& R_{n 1}=\lambda^{2}-\gamma^{2}+\delta \lambda^{2}, \\
& I_{n 1}=-2 \lambda \gamma \zeta \\
& R_{m 1}=(-\mu \delta-\delta-1) \lambda^{4}+\left(\gamma^{2}+\mu \gamma^{2}+1+\delta\right) \lambda^{2}-\gamma^{2}, \\
& I_{m 1}=2 \lambda \gamma \zeta\left(\lambda^{2}-1+\mu \lambda^{2}\right), \\
& R_{n 2}=\delta \lambda\left(\gamma^{2}-\lambda^{2}\right) \\
& I_{n 2}=-2 \gamma \zeta\left(\gamma^{2}-(1+\delta) \lambda^{2}\right), \\
& R_{m 2}=\delta \lambda\left(\lambda^{4}-\left(\gamma^{2}+\mu \gamma^{2}+1\right) \lambda^{2}+\gamma^{2}\right) \text {, } \\
& I_{m 2}=-2 \gamma \zeta\left((1+\delta+\mu \delta) \lambda^{4}-\left(\gamma^{2}+\mu \gamma^{2}+1+\delta\right) \lambda^{2}+\gamma^{2}\right) \text {, } \\
& R_{n 3}=\delta \eta^{2} \gamma \lambda\left(\gamma^{2}-\lambda^{2}\right) \text {, } \\
& I_{n 3}=-2 \zeta\left(\gamma^{4} \eta^{2}-\left(1+\delta \eta^{2}+\eta^{2}\right) \lambda^{2} \gamma^{2}+\lambda^{4}\right), \\
& R_{m 3}=\delta \eta^{2} \gamma \lambda\left(\lambda^{4}-\left(1+\gamma^{2}+\mu \gamma^{2}\right) \lambda^{2}+\gamma^{2}\right) \text {, } \\
& I_{m 3}=2 \zeta\left(\lambda^{6}-\left(1+\mu+\eta^{2}+\delta \eta^{2}+\mu \delta \eta^{2}\right) \lambda^{4}+\left((\mu+1) \eta^{2} \gamma^{2}+1+\eta^{2}+\delta \eta^{2}\right) \gamma^{2} \lambda^{2}-\gamma^{4} \eta^{2},\right. \\
& R_{n 4}=-\delta\left(\lambda^{4}-\left(1+\eta^{2}+\delta \eta^{2}\right) \gamma^{2} \lambda^{2}+\gamma^{4} \eta^{2}\right) \text {, } \\
& I_{n 4}=-2 \gamma \lambda \zeta\left(\gamma^{2}-\lambda^{2}-\delta \lambda^{2}\right) \text {, } \\
& R_{m 4}=\delta\left(\lambda^{6}-\left(1+\left(1+\mu+\eta^{2}+\delta \eta^{2}+\delta \mu \eta^{2}\right) \gamma^{2}\right) \lambda^{4}+\left((\mu+1) \eta^{2} \gamma^{2}+\left(1+\eta^{2}+\delta \eta^{2}\right)\right) \gamma^{2} \eta^{2}-\gamma^{4} \eta^{2}\right) \text {, } \\
& I_{m 4}=-2 \gamma \lambda \zeta\left((1+\delta+\mu \delta) \lambda^{4}-\left(1+\delta+\gamma^{2}+\mu \gamma^{2}\right) \lambda^{2}+\gamma^{2}\right) \text {, } \\
& R_{n 5}=\delta\left(\gamma^{2}-\lambda^{2}\right)\left(\lambda^{2}-\eta^{2} \gamma^{2}\right) \\
& I_{n 5}=-2 \gamma \lambda \zeta\left(\left(1+\delta \eta^{2}\right) \gamma^{2}-(1+\delta) \lambda^{2}\right) \text {, } \\
& R_{m 5}=\delta\left(\lambda^{2}-\eta^{2} \gamma^{2}\right)\left(\lambda^{4}-\left(1+\gamma^{2}+\mu \gamma^{2}\right) \lambda^{2}+\gamma^{2}\right) \text {, } \\
& I_{m 5}=-2 \gamma \lambda \zeta\left((1+\delta+\mu \delta) \lambda^{4}-\left(\left(1+\mu+\delta \eta^{2}+\mu \delta \eta^{2}\right) \gamma^{2}+1+\delta\right) \lambda^{2}+\left(1+\delta \eta^{2}\right) \gamma^{2}\right) \text {, } \\
& R_{n 6}=-\delta\left(\lambda^{4}-\left(1+\eta^{2}+\delta \eta^{2}\right) \gamma^{2} \lambda^{2}+\gamma^{4} \eta^{2}\right), \\
& I_{n 6}=2 \lambda \gamma \zeta\left(\lambda^{2}-\left(1+\delta \eta^{2}\right) \gamma^{2}\right) \text {, } \\
& R_{m 6}=\delta\left(\lambda^{6}-\left(1+\left(1+\mu+\eta^{2}+\delta \eta^{2}+\mu \delta \eta^{2}\right)\right) \lambda^{4}+\left((\mu+1) \eta^{2} \gamma^{2}+\left(1+\eta^{2}+\delta \eta^{2}\right)\right) \gamma^{2} \lambda^{2}-\gamma^{4} \eta^{2}\right), \\
& I_{m 6}=-2 \gamma \lambda \zeta\left(\lambda^{4}-\left(1+\left(1+\mu+\delta \eta^{2}+\mu \delta \eta^{2}\right) \gamma^{2}\right) \lambda^{2}+\left(1+\delta \eta^{2}\right) \gamma^{2}\right) \text {. }
\end{aligned}
$$

\section{Appendix B. Proof of Proposition 1}

From (21), if $C 1$ performs better than the TDVA, that is $I_{C 1}<I_{T D V A}$, the second term of (21) must be less than 0 , which means

$$
\delta^{2}+a_{C 1,1} \delta<0
$$

Since $\delta \geq 0$, if $\gamma^{2}<\frac{1}{1+\mu}$, the optimal $\delta$ denoted as $\delta_{\text {opt }}$ is 0 . If $\gamma^{2} \geq \frac{1}{1+\mu}$, the optimal $\delta_{\text {opt }}=(1+\mu) \gamma^{2}-1$, and it can be checked that the optimal $\gamma$ is $\frac{1}{1+\mu}$ by substituting $\delta_{\text {opt }}$ into (21), which means that the optimal $\delta$ is also 0 . 


\section{Appendix C. Proof of Proposition 2}

First, we prove that $C 2$ performs better than the TDVA, that is $I_{C 2, \text { opt }}<I_{T D V A, \text { opt }}$, where $I_{C 2, \text { opt }}$ denotes the optimal $I_{C 2}$. From (23), if $C 2$ performs better than the TDVA, the following inequality must hold

$$
a_{C 2,2} \delta^{-2}+a_{C 2,1} \delta^{-1}<0,
$$

which requires that

$$
a_{C 2,1}<0 \text { or } \gamma^{2}>\frac{2+\mu}{2(1+\mu)^{2}},
$$

as $a_{C 2,2} \geq 0$ for any $\gamma \geq 0$. If $\gamma^{2}>\frac{2+\mu}{2(1+\mu)^{2}}$, the optimal $\delta^{-1}$ is

$$
\delta_{\text {opt }}^{-1}=-\frac{a_{C 2,1}}{2 a_{C 2,2}},
$$

and $I_{C 2}$ can be represented as

$$
I_{C 2}=\sqrt{\frac{\left(1-(2+\mu) \gamma^{2}+(1+\mu)^{2} \gamma^{4}\right)\left(4(1+\mu)^{2} \gamma^{2}-\mu\right)}{4 \mu\left(1-2(1+\mu) \gamma^{2}+(1+\mu)^{3} \gamma^{4}\right)}} .
$$

Using $I_{T D V A, \text { opt }}$ given in (16), one obtains

$$
I_{C 2}^{2}-I_{T D V A, \text { opt }}^{2}=\frac{\left((\mu+1) \gamma^{2}-1\right)\left(2(\mu+1)^{2} \gamma^{2}-2-\mu\right)^{2}}{4 \mu\left(1-2(\mu+1) \gamma^{2}+(\mu+1)^{3} \gamma^{4}\right)(\mu+1)},
$$

Clearly, if $\gamma^{2}<\frac{1}{1+\mu}$, then $I_{C 2}<I_{T D V A, o p t}$. Since $\frac{1}{1+\mu}>\frac{2+\mu}{2(1+\mu)^{2}}$, one can always find a $\gamma$ such that $I_{C 2}<I_{T D V A, \text { opt }}$. Since $I_{C 2, \text { opt }} \leq I_{C 2}$, one obtains $I_{C 2, \text { opt }}<I_{T D V A, \text { opt }}$.

Second, we graphically prove that only at most $0.32 \%$ improvement can be obtained by $C 2$ when $\mu \leq 1$. The optimal $\gamma$ can be obtained by solving $\frac{\partial I_{C 2}^{2}}{\partial \gamma^{2}}=0$, which is equivalent to

$$
\left(2 \alpha^{2} \gamma^{2}-1-\alpha\right)\left(2 \alpha^{5} \gamma^{6}+\left(\alpha^{4}-7 \alpha^{3}\right) \gamma^{4}+\left(8 \alpha^{2}-2 \alpha^{3}\right) \gamma^{2}-3 \alpha+1\right)=0,
$$

where $\alpha=\mu+1$. It is easy to check that (C.2) has two real positive solutions denoted as $\gamma_{1}$ and $\gamma_{2}, \gamma_{1}<\gamma_{2}$, where

$$
\gamma_{1}=\sqrt{\frac{1+\alpha}{2 \alpha^{2}}}
$$

and the optimal $\gamma$ is $\gamma_{2}$.

For $0 \leq \mu \leq 1$, a graphical comparison with the TDVA is shown in Fig. C.12, where it is clearly shown that at most $0.32 \%$ improvement is obtained for $C 2$. 


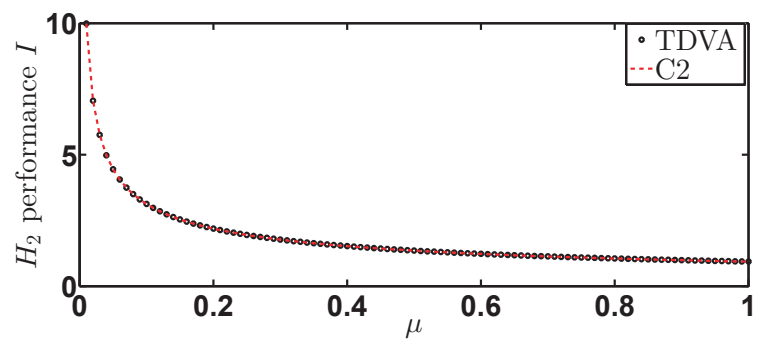

(a)

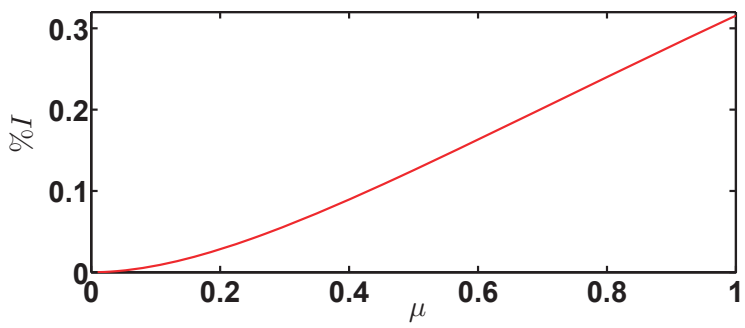

(b)

Figure C.12: Comparison between $C 2$ and TDVA when $0 \leq \mu \leq 1$. (a) the $H_{2}$ performance; (b) Percentage improvement of $C 2$ with respect to TDVA.

\section{Appendix D. Analytical representations of the $\mathrm{H}_{2}$ performance measures for $C 3, C 4, C 5$, and $C 6$}

Denote $I_{C 3}, I_{C 4}, I_{C 5}$, and $I_{C 6}$ as the $H_{2}$ performance measures for $C 3, C 4, C 5$, and $C 6$, respectively. The detailed representations are obtained as follows:

$$
\begin{aligned}
I_{C 3} & =\left(a_{C 3,2} \delta^{-2}+a_{C 3,1} \delta^{-1}+a_{C 3,0}\right) \zeta+\frac{1-(\mu+2) \gamma^{2}+(1+\mu)^{2} \gamma^{4}}{4 \gamma \mu \zeta} \\
& =I_{T D V A}+\left(a_{C 3,2} \delta^{-2}+a_{C 3,1} \delta^{-1}\right) \zeta \\
I_{C 4} & =\left(a_{C 4,2} \delta^{-2}+a_{C 4,1} \delta^{-1}+a_{C 4,0}\right) \zeta+\left(l_{C 4,2} \eta^{4} \delta^{2}+l_{C 4,1} \delta+l_{C 4,0}\right) \frac{1}{\zeta} \\
& =I_{T D V A}+\left(a_{C 4,2} \delta^{-2}+a_{C 4,1} \delta^{-1}\right) \zeta+\left(l_{C 4,2} \eta^{4} \delta^{2}+l_{C 4,1} \delta+f_{C 4,2} \eta^{4}+f_{C 4,1} \eta^{2}\right) \frac{1}{\zeta} \\
I_{C 5} & =\left(a_{C 5,2} \delta^{-2}+a_{C 5,1} \delta^{-1}+a_{C 5,0}\right) \zeta+\frac{1}{4 \gamma \mu \zeta}\left(1-(\mu+2) \gamma^{2}+(1+\mu)^{2} \gamma^{4}\right) \\
& =I_{T D V A}+\left(a_{C 5,2} \delta^{-2}+a_{C 5,1} \delta^{-1}\right) \zeta \\
I_{C 6} & =\left(a_{C 6,2} \delta^{-2} \eta^{-4}+a_{C 6,1} \delta^{-1} \eta^{-2}+a_{C 6,0}\right) \zeta+\left(l_{C 6,2} \delta^{2}+l_{C 6,1} \delta+l_{C 6,0}\right) \frac{1}{\zeta} \\
& =I_{T D V A}+\left(a_{C 6,2} \delta^{-2} \eta^{-4}+a_{C 6,1} \delta^{-1} \eta^{-2}\right) \zeta+\left(l_{C 6,2} \delta^{2}+l_{C 6,1} \delta+f_{C 6,2} \eta^{-4}+f_{C 6,1} \eta^{-2}\right) \frac{1}{\zeta}
\end{aligned}
$$


where

$$
\begin{aligned}
& a_{C 3,2}=d_{C 3,2} \eta^{-4}+d_{C 3,1} \eta^{-2}+d_{C 3,0}, a_{C 3,1}=g_{C 3,1} \eta^{-2}+g_{C 3,0}, a_{C 3,0}=\frac{\gamma(1+\mu)}{\mu}, \\
& d_{C 3,2}=\frac{1}{\gamma^{3} \mu}\left(1-2 \gamma^{2}+(1+\mu) \gamma^{4}\right), d_{C 3,1}=-\frac{2}{\gamma \mu}\left(1-(2+\mu) \gamma^{2}+(1+\mu)^{2} \gamma^{4}\right), \\
& d_{C 3,0}=\frac{\gamma}{\mu}\left(1-2(1+\mu) \gamma^{2}+(1+\mu)^{3} \gamma^{4}\right), g_{C 3,1}=-\frac{2}{\mu \gamma}\left(1-(1+\mu) \gamma^{2}\right), \\
& g_{C 3,0}=-\frac{\gamma}{\mu}\left(2(1+\mu)^{2} \gamma^{2}-2-\mu\right) \text {, } \\
& a_{C 4,2}=\frac{\gamma}{\mu}\left(1-(2+\mu) \gamma^{2}+(1+\mu)^{3} \gamma^{4}\right), a_{C 4,1}=\frac{\gamma}{\mu}\left(2+\mu-2(1+\mu)^{2} \gamma^{2}\right), a_{C 4,0}=\frac{\gamma(1+\mu)}{\mu}, \\
& l_{C 4,2}=\frac{\gamma^{3}(1+\mu)^{2}}{4 \mu}, l_{C 4,1}=g_{C 4,2} \eta^{4}+g_{C 4,1} \eta^{2}, l_{C 4,0}=f_{C 4,2} \eta^{4}+f_{C 4,1} \eta^{2}+f_{C 4,0}, \\
& g_{C 4,2}=\frac{\gamma^{3}}{2 \mu}\left(1+\mu-(1+\mu)^{3} \gamma^{2}\right), g_{C 4,1}=\frac{\gamma}{4 \mu}\left(2(1+\mu)^{2} \gamma^{2}-\mu-2\right) \text {, } \\
& f_{C 4,2}=\frac{\gamma^{3}}{4 \mu}\left((1+\mu)^{4} \gamma^{4}+(\mu-2)(\mu+1)^{2} \gamma^{2}+1\right), f_{C 4,1}=-\frac{\gamma}{2 \mu}\left((1+\mu)^{3} \gamma^{4}-2(1+\mu) \gamma^{2}+1\right) \text {, } \\
& f_{C 4,0}=\frac{1}{4 \mu \gamma}\left(1-(\mu+2) \gamma^{2}+(1+\mu)^{2} \gamma^{4}\right) \text {, } \\
& a_{C 5,2}=\frac{g_{C 5,2} \eta^{4}+g_{C 5,1} \eta^{2}+g_{C 5,0}}{\mu\left(1+f_{C 5,1} \eta^{2}+f_{C 5,2} \eta^{4}\right)^{2}}, a_{C 5,1}=\frac{l_{C 5,3} \eta^{6}+l_{C 5,2} \eta^{4}+l_{C 5,1} \eta^{2}+l_{C 5,0}}{\mu\left(1+f_{C 5,1} \eta^{2}+f_{C 5,2} \eta^{4}\right)^{2}}, a_{C 5,0}=\frac{\gamma(1+\mu)}{\mu}, \\
& g_{C 5,2}=\gamma\left((1+\mu) \gamma^{4}-2 \gamma^{2}+1\right), g_{C 5,1}=-2 \gamma\left((1+\mu)^{2} \gamma^{4}-(\mu+2) \gamma^{2}+1\right) \text {, } \\
& g_{C 5,0}=\gamma\left((1+\mu)^{3} \gamma^{4}-2(1+\mu) \gamma^{2}+1\right), f_{C 5,1}=-\left(1+\gamma^{2}(1+\mu)\right), f_{C 5,2}=\gamma^{2} \text {, } \\
& l_{C 5,3}=2 \gamma^{3}\left((1+\mu)^{3}-1\right), l_{C 5,2}=-\gamma\left(4(1+\mu)^{2} \gamma^{4}-2 \gamma^{2}-\mu-2\right), \\
& l_{C 5,1}=2 \gamma\left((1+\mu)^{3} \gamma^{4}+(1+\mu)^{2} \gamma^{2}-\mu-2\right), l_{C 5,0}=\gamma\left(\mu+2-2(1+\mu)^{2} \gamma^{2}\right) \text {, } \\
& a_{C 6,2}=\frac{1-2 \gamma^{2}+(1+\mu) \gamma^{4}}{\gamma^{3} \mu}, a_{C 6,1}=\frac{2\left((1+\mu) \gamma^{2}-1\right)}{\gamma \mu}, a_{C 6,0}=\frac{\gamma(1+\mu)}{\mu} \text {, } \\
& l_{C 6,2}=\frac{1}{4 \gamma \mu}, l_{C 6,1}=g_{C 6,1} \eta^{-2}+g_{C 6,0}, l_{C 6,0}=f_{C 6,2} \eta^{-4}+f_{C 6,1} \eta^{-2}+f_{C 6,0}, \\
& g_{C 6,1}=\frac{\mu-2+2 \gamma^{2}}{4 \gamma^{3} \mu}, g_{C 6,0}=\frac{1-(1+\mu) \gamma^{2}}{2 \gamma \mu} \text {, } \\
& f_{C 6,2}=\frac{1+(\mu-2) \gamma^{2}+\gamma^{4}}{4 \mu \gamma^{5}}, f_{C 6,1}=-\frac{1-2 \gamma^{2}+(1+\mu) \gamma^{4}}{2 \mu \gamma^{3}}, f_{C 6,0}=\frac{1-(2+\mu) \gamma^{2}+(1+\mu)^{2} \gamma^{4}}{4 \gamma \mu} \text {. }
\end{aligned}
$$

\section{Appendix E. Proof of Proposition 3}

For $C 3$, substituting $\gamma_{T D V A, o p t}$ and $\zeta_{T D V A, o p t}$ into (D.1), one obtains

$$
I_{C 3}^{\prime}=I_{T D V A, o p t}+\left(a_{C 3,2}^{\prime} \delta^{-2}+a_{C 3,1}^{\prime} \delta^{-1}\right) \zeta_{T D V A, o p t},
$$


330

$$
a_{C 3,1}^{\prime}=-\sqrt{\frac{2}{2+\mu}} \eta^{-2}<0
$$

which means that there exist finite $\delta$ and $\eta$ such that $I_{C 3}^{\prime}<I_{T D V A, o p t}$. Since $I_{C 3, \text { opt }} \leq I_{C 3}^{\prime}$, then one obtains $I_{C 3, \text { opt }}<I_{T D V A, \text { opt }}$.

For $C 4$, denote

$$
I_{C 4}^{\prime}=2 \sqrt{\left(a_{C 4,2}^{\prime} \delta^{-2}+a_{C 4,1}^{\prime} \delta^{-1}+a_{C 4,0}^{\prime}\right)\left(l_{C 4,2}^{\prime} \eta^{4} \delta^{2}+l_{C 4,1}^{\prime} \delta+l_{C 4,0}^{\prime}\right)},
$$

where $a_{C 4,2}^{\prime}, a_{C 4,1}^{\prime}, a_{C 4,0}^{\prime}, l_{C 4,2}^{\prime}, l_{C 4,2}^{\prime}$, and $l_{C 4,0}^{\prime}$ are obtained by setting $\gamma=\gamma_{T D V A, o p t}$.

Expanding $I_{C 4}^{\prime}$, one obtains

$$
I_{C 4}^{\prime}=2 \sqrt{a_{C 4,0}^{\prime} f_{C 4,0}^{\prime}+f_{C 4, \eta}},
$$

where

$f_{C 4, \eta}=\left(l_{C 4,2}^{\prime} \delta^{2}+g_{C 4,2}^{\prime} \delta+f_{C 4,2}^{\prime}\right)\left(a_{C 4,2}^{\prime} \delta^{-2}+a_{C 4,0}^{\prime}\right) \eta^{4}+f_{C 4,1}^{\prime}\left(a_{C 4,2}^{\prime} \delta^{-2}+a_{C 4,0}^{\prime}\right) \eta^{2}+f_{C 4,0}^{\prime} a_{C 4,2}^{\prime} \delta^{-2}$.

Note that

$$
I_{T D V A, o p t}=2 \sqrt{a_{C 4,0}^{\prime} f_{C 4,0}^{\prime}} .
$$

Then, we will prove that there exist finite $\delta$ and $\eta$ so that $f_{C 4, \eta}<0$. It can be checked that $l_{C 4,2}^{\prime} \delta^{2}+g_{C 4,2}^{\prime} \delta+f_{C 4,2}^{\prime}>0, a_{C 4,2}^{\prime} \delta^{-2}+a_{C 4,0}^{\prime}>0$, and $f_{C 4,1}^{\prime}\left(a_{C 4,2}^{\prime} \delta^{-2}+a_{C 4,0}^{\prime}\right)<0$. The discriminant of $f_{C 4, \eta}=0$ is

$$
\Delta=\left(a_{C 4,2}^{\prime} \delta^{2}+a_{C 4,0}^{\prime}\right)\left(\left(f_{C 4,1}^{\prime}{ }^{2}-4 f_{C 4,2}^{\prime} f_{C 4,0}^{\prime}\right) a_{C 4,2}^{\prime} \delta^{-2}-4 g_{C 4,2}^{\prime} f_{C 4,0}^{\prime} a_{C 4,2}^{\prime} \delta^{-1}+\right.
$$

$$
\left.f_{C 4,1}^{\prime}{ }^{2} a_{C 4,0}^{\prime}-4 l_{C 4,2}^{\prime} f_{C 4,0}^{\prime} a_{C 4,2}^{\prime}\right) .
$$

It can be checked that if $\mu<\frac{8 \sqrt{2}-4}{7} \approx 1.045$, there exists a finite $\delta$ such that the second term of $\Delta$ is positive, which means that if $\mu<1.045$, there exists a finite $\eta$ such that $f_{C 4, \eta}<0$. For example, if choosing

$$
\delta^{-1}=\frac{2 g_{C 4,2}^{\prime} f_{C 4,0}^{\prime}}{f_{C 4,1}^{\prime}-4 f_{C 4,2}^{\prime} f_{C 4,0}^{\prime}}=\frac{(3 \mu+4)(1+\mu)}{4 \mu(\mu+2)},
$$

and

$$
\eta=\sqrt{\frac{-f_{C 4,1}^{\prime}}{l_{C 4,2}^{\prime} \delta^{2}+g_{C 4,2}^{\prime} \delta+f_{C 4,2}^{\prime}}}=\sqrt{\frac{2(3 \mu+4)^{2}(1+\mu)(4+\mu)}{(\mu+2)\left(43 \mu^{3}+204 \mu^{2}+272 \mu+64\right)}},
$$

one obtains

$$
f_{C 4, \eta}=\frac{1}{128} \frac{\left(7 \mu^{2}+8 \mu-16\right)(\mu+4)(3 \mu+4)^{2}}{\mu\left(43 \mu^{3}+204 \mu^{2}+272 \mu+64\right)(1+\mu)(\mu+2)}<0 .
$$


From (E.1) and for the $\delta$ and $\eta$ given by (E.2) and (E.3), one obtains that if $\mu<1.045$,

$$
I_{C 4}^{\prime}<I_{T D V A, o p t}
$$

342

Since $I_{C 4, \text { opt }} \leq I_{C 4}^{\prime}$, one obtains that if $\mu<1.045, I_{C 4, \text { opt }}<I_{T D V A, \text { opt }}$.

For $C 5$, setting $\gamma=\gamma_{T D V A, o p t}$ and $\zeta=\zeta_{T D V A, \text { opt }}$ in (D.2), one obtains

$$
I_{C 5}^{\prime}=I_{T D V A, o p t}+\left(a_{C 5,2}^{\prime} \delta^{-2}+a_{C 5,1}^{\prime} \delta^{-1}\right) \zeta_{T D V A, o p t} .
$$

Then, we will show that there exist finite $\delta$ and $\eta$ such that $a_{C 5,2}^{\prime} \delta^{-2}+a_{C 5,1}^{\prime} \delta^{-1}<0$. It can be checked that $a_{C 5,2}^{\prime}>0$. Therefore, we only need to prove that there exists a finite $\eta$ such that $a_{C 5,1}^{\prime}<0$. Since

$$
a_{C 5,1}^{\prime}=\frac{l_{C 5,3}^{\prime} \eta^{6}+l_{C 5,2}^{\prime} \eta^{4}+l_{C 5,1}^{\prime} \eta^{2}}{\mu\left(1+f_{C 5,1}^{\prime} \eta^{2}+f_{C 5,2}^{\prime} \eta^{4}\right)^{2}}
$$

one obtains

$$
f_{\delta}=-\frac{\sqrt{2}(2+\mu)^{5 / 2}(\mu+1)^{2}}{\left(1+8 \mu+4 \mu^{2}\right)\left(4+9 \mu+4 \mu^{2}\right)\left(1+3 \mu+5 \mu^{2}+2 \mu^{3}\right)^{2}}<0,
$$

which means that for the $\eta$ and $\delta$ given by (E.5) and (E.6), $I_{C 5}^{\prime}<I_{T D V A, \text { opt }}$. Since $I_{C 5, \text { opt }} \leq$ $I_{C 5}^{\prime}$, one obtains $I_{C 5, \text { opt }}<I_{T D V A, o p t}$.

For $C 6$, setting $\gamma=\gamma_{T D V A, o p t}$ and $\zeta=\zeta_{T D V A, o p t}$, one obtains

$$
I_{C 6}^{\prime}=I_{T D V A, o p t}+f_{C 6, \eta}
$$

where $f_{C 6, \eta}=d_{2} \eta^{-4}+d_{1} \eta^{-2}+d_{0}$, with

$$
\begin{aligned}
d_{2} & =a_{C 6,2}^{\prime} \zeta_{T D V A, o p t} \delta^{-2}+f_{C 6,2}^{\prime} / \zeta_{T D V A, o p t}, \\
d_{1} & =a_{C 6,1}^{\prime} \zeta_{T D V A, o p t} \delta^{-1}+\left(g_{C 6,1}^{\prime} \delta+f_{C 6,1}^{\prime}\right) / \zeta_{T D V A, o p t}, \\
d_{0} & =\left(l_{C 6,2}^{\prime} \delta^{2}+g_{C 6,0}^{\prime} \delta\right) / \zeta_{T D V A, o p t} .
\end{aligned}
$$

It can be checked that $d_{2}>0$ for any $\delta$ and if $\mu<\sqrt{2}, d_{1}<0$. Thus, it remains to prove that there exists a finite $\eta>0$ such that $f_{C 6, \eta}<0$. This can be done by checking the discriminant of $f_{C 6, \eta}$, which is

$$
\begin{aligned}
\Delta= & d_{1}^{2}-4 d_{2} d_{0} \\
= & 16(\mu-4)(\mu+1)^{8} \delta^{4}-16 \mu\left(4 \mu^{3}+11 \mu^{2}+5 \mu-4\right)(\mu+1)^{4} \delta^{3}+ \\
& 8 \mu^{2}\left(5 \mu^{2}+21 \mu+20\right)(\mu+1)^{3} \delta^{2}+\mu^{3}(3 \mu+4)^{2} .
\end{aligned}
$$


It is easy to see that there always exists a finite $\delta$ such that $\Delta>0$. For example, if choosing

$$
\delta=\frac{\mu\left(4 \mu^{3}+11 \mu^{2}+5 \mu-4-\sqrt{6 \mu^{6}+56 \mu^{5}+253 \mu^{4}+606 \mu^{3}+799 \mu^{2}+568 \mu+176}\right)}{2(\mu-4)(\mu+1)^{4}},
$$

which is larger than 0 if $\mu<4$, one obtains

$$
\Delta=\mu^{3}(3 \mu+4)^{2}>0 .
$$

Therefore, we can always find a $\eta^{-2}$ between the two real positive solutions of $f_{C 6, \eta}=0$ such that $f_{C 6, \eta}<0$. A possible choice is $\eta^{-2}=-\frac{d_{1}}{2 d_{2}}$. This means that if carefully choosing $\delta$ and $\eta$, the inequality $I_{C 6}^{\prime}<I_{I D V A, \text { opt }}$ holds. Since $I_{C 6, \text { opt }} \leq I_{C 6}^{\prime}$, one obtains $I_{C 6, \text { opt }}<I_{T D V A, o p t}$.

\section{Acknowledgment}

The authors are grateful to the Associate Editor and the reviewers for their insightful suggestions.

This research was partially supported by the Research Grants Council, Hong Kong, through the General Research Fund under Grant 17200914, the Innovation and Technology Commission under Grant ITS/178/13, and the Natural Science Foundation of China under Grants 61374053.

\section{References}

[1] Den Hartog JP. Mechanical Vibrations. New York: Courier Dover Publications; 1985.

[2] Frahm H. Device for damping vibrations of bodies. US Patent, No. 989958. 30 October 1909.

[3] Ormondroyd J, Den Hartog JP. The theory of the dynamic vibration absorber. ASME Journal of Applied Mechanics 1928;50:9-22.

[4] Nishihara O, Asami T. Closed-form solutions to the exact optimizations of dynamic vibration absorbers (minimizations of the maximum amplitude magnification factors). Journal of Vibration and Acoustics 2002;124(4):576-82.

[5] Crandall SH, Mark WD. Random Vibration in Mechanical Systems. New York: Academic Press; 1963.

[6] Asami T, Wakasono T, Kameoka K, Hasegawa M, Sekiguchi H. Optimum design of dynamic absorbers for a system subjected to random excitation. JSME International Journal Ser. 3, Vibration, Control Engineering, Engineering for Industry 1991;34(2):21826.

[7] Anh ND, Nguyen NX. Design of TMD for damped linear strucutures using the dual criterion of equivalent linearization method. International Journal of Mehcanical Sciences 2013;77:164-70. 
[8] Ghosh A, Basu B. A closed-form optimal tuning criteiron for TMD in damped structures. Structural Control and Health Monitoring 2007;14:681-92.

[9] Asami T, Nishihara O, Baz AM. Analytical solutions to $H_{\infty}$ and $H_{2}$ optimization of dynamic vibration absorbers attached to damped linear systems. Journal of Vibration and Acoustics 2002;124(2):284-95.

[10] Bekdas G, Nigdeli SM. Mass ratio factor for optimum tuned mass damper strategies. International Journal of Mechanical Sciences 2013;71:68-84.

[11] Cheung YL, Wong WO. $H_{\infty}$ and $H_{2}$ optimizations of a dyanmic vibration absorber for suppressing vibrations in plates. Journal of Sound and Vibration 2009;320:29-42.

[12] Pai PF, Schulz MJ. A refined nonlinear vibration absorber. International Journal of Mechanical Sciences 2000;42(3):537-60.

[13] Miguelez MH, Rubio L, Loya JA, Fernandez-Saez J. Improvement of chatter stability in boring operations with passive vibration absorbers. International Journal of Mechanical Sciences 2010;52(10):1376-84.

[14] Gao H, Zhan W, Karimi HR, Yang X, Yin S. Allocation of actuators and sensors for coupled-adjacent-building vibration attenuation. IEEE Transactions on Industrial Electronics 2013;60(12):5792-801.

[15] Si Y, Karimi HR, Gao H. Modelling and optimization of a passive structural control design for a spar-type floating wind turbine. Engineering Structures 2014;69:168-82.

[16] Zhan W, Cui Y, Feng Z, Cheung KC, Lam J, Gao H. Joint optimization approach to building vibration control via multiple active tuned mass dampers. Mechatronics 2013;23(3):355-68.

[17] Smith MC. Synthesis of mechanical networks: the inerter. IEEE Trans Autom Control 2002;47(10):1648-62.

[18] Chen MZQ, Papageorgiou C, Scheibe F, Wang FC, Smith MC. The missing mechanical circuit element. IEEE Circuits Syst Mag 2009;9(1):10-26.

[19] Smith MC, Wang FC. Performance benefits in passive vehicle suspensions employing inerters. Veh Syst Dyn 2004;42(4):235-57.

[20] Chen MZQ, Hu Y, Du B. Suspension performance with one damper and one inerter. The 24th Chinese Control and Decision Conference. Taiyuan: China;2012, p. 3551-3556.

[21] Chen MZQ, Hu Y, Li C, Chen G. Performance benefits of using inerter in semiactive suspensions. IEEE Trans Control Syst Technol, in press. DOI:10.1109/TCST.2014.2364954.

[22] Hu Y, Chen MZQ, Shu Z. Passive vehicle suspensions employing inerters with multiple performance requirements. Journal of Sound and Vibration 2014;333(8):2212-25. 
[23] Lazar IF, Neild SA, Wagg DJ. Using an inerter-based device for structural vibration suppression. Earthquake Engng Struct Dyn 2014;43(8):1129-47.

[24] Hu Y, Chen MZQ, Shu Z, Huang L. Analysis and optimisation for inerter-based isolators via fixed-point theory and algebraic solution. Journal of Sound and Vibration $2015 ; 346: 17-36$.

[25] Marian L, Giaralis, A. Optimal design of a novel tuned mass-damper-inerter(TMDI) passive vibration control configuration for stochastically support-excited structural systems. Probabilistic Engineering Mechanics 2014;38:156-64.

[26] Brzeski P, Pavlovskaia E, Kapitaniak T, Perlikowski P. The application of inerter in tuned mass absorber. International Journal of Non-Linear Mechanics 2015;70:20-9.

[27] Chen MZQ, Hu Y, Huang L, Chen G. Influence of inerter on natural frequencies of vibration systems. Journal of Sound and Vibration 2014;333(7):1874-87.

[28] Chen MZQ, Smith MC. Electrical and mechanical passive network synthesis. In Recent Advances in Learning and Control, New York: Springer-Verlag; 2008;371:35-50.

[29] Chen MZQ, Smith MC. A note on tests for positive-real functions. IEEE Trans Autom Control 2009;54(2):390-3.

[30] Chen MZQ. A note on PIN polynomials and PRIN rational functions. IEEE Transactions on Circuits and Systems II: Express Briefs 2008;55(5):462-3.

[31] Chen MZQ, Smith MC. Restricted complexity network realizations for passive mechanical control. IEEE Trans Autom Control 2009;54(10):2290-301.

[32] Wang K, Chen MZQ. Generalized series-parallel RLC synthesis without minimization for biquadratic impedances. IEEE Trans on Circuits and Systems II: Express Briefs 2012;59(11):766-70.

[33] Chen MZQ, Wang K, Zou Y, Lam J. Realization of a special class of admittances with one damper and one inerter for mechanical control. IEEE Trans Autom Control 2013;58(7):1841-46.

[34] Chen MZQ, Wang K, Shu Z, Li C. Realizations of a special class of admittances with strictly lower complexity than canonical forms. IEEE Trans Circuits and Systems-I: Regular Papers 2013;60(9):2465-73.

[35] Chen MZQ, Wang K, Yin M, Li C, Zuo Z, Chen G. Synthesis of $n$-port resistive networks containing $2 n$ terminals. International Journal of Circuit Theory \& Applications $2015 ; 43(4): 427-437$.

[36] Shearer JL, Murphy AT, Richardson HH. Introduction to System Dynamics. Reading, MA: Addison-Wesley; 1967.

[37] Hixson EL. Mechanical impedance. In: Harris CM, editor. Shock and Vibration Handbook, 3rd ed, New York: McGraw-Hill; 1988, ch. 10. 
[38] Wang K, Chen MZQ, Hu Y. Synthesis of biquadratic impedances with at most four passive elements. Journal of the Franklin Institute 2014;351(3):1251-67.

[39] Asami T, Wakasono T, Kameoka K, Hasegawa M, Sekiguchi H. Optimum design of dynamic absorbers for a system subjected to random excitation. JSME International Journal Series III 1991;34(2):218-26.

[40] Cheung YL, Wong WO. $\mathrm{H}_{2}$ optimization of a non-traditional dynamic vibration abosorber for vibration control of structures under random force excitation. Journal of Sound and Vibration 2011;330(6):1039-44.

[41] Doyle JC, Francis BA, Tannenbaum AR. Feedback Control Theory. Oxford: Maxwell Macmillan Int; 1992.

[42] Inman DJ. Engineering Vibration. 3rd ed. NJ: Prentice-Hall; 2008.

[43] Cheung YL, Wong WO. H-infinity optimization of a variant design of the dynamic vibration absorber-Revisited and new results. Journal of Sound and Vibration 2011;330:390112 . 